\title{
Hebbian plasticity in parallel synaptic pathways: A circuit mechanism for systems memory consolidation
}

Michiel Remme ${ }^{1 \dagger}$, Urs Bergmann ${ }^{1 \dagger}$, Denis Alevi ${ }^{2,3}$, Susanne Schreiber ${ }^{1,3,4}$, Henning

Sprekeler ${ }^{2,3,4,5+}$, Richard Kempter ${ }^{1,3,4,+, *}$

${ }^{1}$ Department of Biology, Institute for Theoretical Biology, Humboldt-Universität zu

Berlin, Phillipstr. 13, 10115 Berlin, Germany

${ }^{2}$ Department for Electrical Engineering and Computer Science, Technische Universität

Berlin, Marchstr. 23, 10587 Berlin, Germany

3 Bernstein Center for Computational Neuroscience Berlin, Philippstr. 13, 10115 Berlin, Germany

${ }^{4}$ Einstein Center for Neurosciences Berlin, Charitéplatz 1, 10117 Berlin, Germany

${ }^{5}$ Excellence Cluster Science of Intelligence, Marchstr. 23, 10587 Berlin, Germany

* r.kempter@biologie.hu-berlin.de

$\dagger,+$ equal contribution 


\section{Abstract}

Systems memory consolidation involves a transfer of declarative memories that initially depend on the hippocampal formation into long-term memory traces in neocortical networks. This consolidation process is thought to rely on replay of recently acquired memories, but the cellular and network mechanisms that mediate the memory transfer are poorly understood. Here, we suggest that systems memory consolidation could arise from Hebbian plasticity in networks with parallel synaptic pathways - two ubiquitous features of neural circuits in the brain. We explore this hypothesis in a computational model to illustrate how memories are transferred across circuits and why their representations could change. These modelling results are in quantitative agreement with lesion studies in rodents. A hierarchical iteration of the mechanism yields power-law forgetting — as observed in psychophysical studies in humans. The predicted circuit mechanism thus bridges spatial scales from single cells to cortical areas and time scales from milliseconds to years.

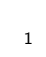




\section{Introduction}

Clinical and lesion studies suggest that declarative memories initially depend on the

hippocampus, but are later transferred to other brain areas (Dudai et al. 2015: Squire

independent of the hippocampus and depend only on a stable representation in the neocortex (Dudai et al. 2015, Squire et al. 2015, Sekeres et al., 2017). This process termed systems memory consolidation — is thought to prevent newly acquired memories from overwriting old ones, thereby extending memory retention times ("plasticity-stability dilemma"; Grossberg (1987); Abraham and Robins (2005); Fusi et al. (2005); Leibold and Kempter (2008); Roxin and Fusi (2013)), and to enable a simultaneous acquisition of episodic memories and semantic knowledge of the world (McClelland et al., 1995, Kumaran et al., 2016). While specific neuronal activity patterns, including for example an accelerated replay of recent experiences Lee and Wilson, 2002, Skaggs and McNaughton, 1996), are involved in the transfer of memories from hippocampus to neocortex (Diekelmann and Born, 2010), the mechanisms underlying systems memory consolidation are not well understood. Specifically, it is unclear how this consolidation is shaped by the anatomical structure and the plasticity of the underlying neural circuits. This poses a substantial obstacle for understanding into which regions memories are consolidated; why some memories are consolidated more rapidly than others (Nadel and Moscovitch, 1997; Tse et al. 2007 ; Brodt et al. 2018); why some memories stay hippocampus dependent, and why and how the character of memories changes over time (Dudai et al. 2015$)$; and whether the consolidation of declarative and non-declarative memories (Karni et al. 1994 , Brashers-Krug et al. 1996 , Dudai et al. 2015) are two sides of the same coin. These questions are hard to approach within phenomenological theories of systems consolidation such as the standard consolidation theory (Squire and Alvarez, 1995 . McClelland et al. 1995), the multiple trace theory (Nadel and Moscovitch, 1997), and the trace transformation theory (Winocur et al. 2010, Winocur and Moscovitch, 2011). Here, we propose a mechanistic foundation of the consolidation process that accounts for several experimental observations and could contribute to understanding the transfer of memories and the reorganisation of memories over time on a neuronal level. 
Our focus lies on simple forms of memory that can be phrased as cue-response associations. We assume that such associations are stored in synaptic pathways between an input area — neurally representing thec cue — and an output area — neurally representing the response. Our central hypothesis - the parallel pathway theory (PPT)

- is that systems memory consolidation arises naturally from the interplay of two abundantly found neuronal features: parallel synaptic pathways and Hebbian plasticity (Van Essen et al. 1992, Malenka and Bear, 2004). First, we illustrate this theory in a simple hippocampal circuit motif and show that Hebbian plasticity can consolidate previously stored associations into parallel pathways. The mechanism reproduces the results of a hippocampal lesion study in rodents (Remondes and Schuman, 2004). Iterated in a cascade, it can achieve a full consolidation into neocortex and result in power-law forgetting of memories as is observed in psychophysical studies in humans Wixted, 2004).

\section{Results}

\section{A mechanistic basis for systems memory consolidation}

The suggested parallel pathway theory (PPT) relies on a parallel structure of feedforward connections onto the same output area: a direct, monosynaptic and an indirect, multisynaptic pathway. We propose that memories are initially stored in the indirect pathway and are subsequently transferred to the direct pathway via Hebbian plasticity. Because the indirect pathway is multisynaptic, it transmits signals with a longer time delay than the direct pathway (Fig. 1A). This allows the indirect pathway to act as a teacher for the direct pathway. The mechanism can be exemplified in the hippocampal formation Amaral, 1993) as follows. We assume that CA3 is involved in the original storage of declarative memories $($ Marr, 1969, Treves and Rolls, 1994. Nakazawa et al. 2003). The indirect pathway then corresponds to the classical trisynaptic pathway from entorhinal cortex (EC) through dentate gyrus (DG) and CA3, reaching CA1 through the Schaffer collaterals (SC; Fig. 1B, blue; Amaral (1993)). A memory can be recalled by a specific neural activity pattern in EC - a cue - that triggers spikes in a subset of CA1 cells through this indirect pathway, representing the
47 
A

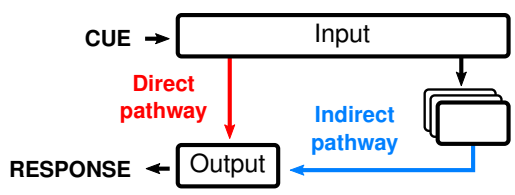

B

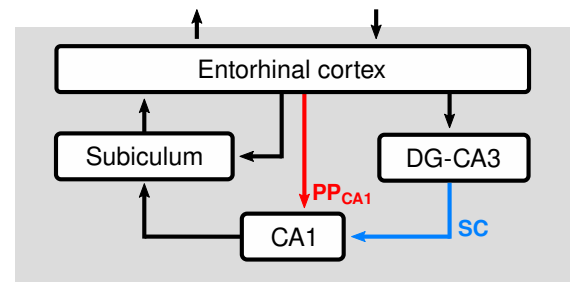

D

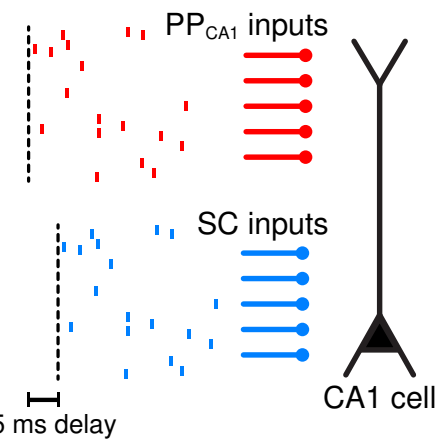

E

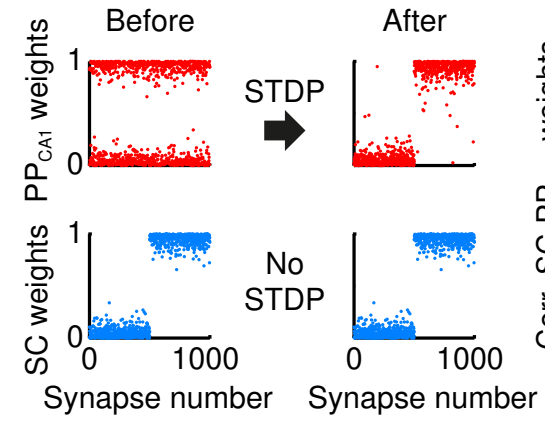

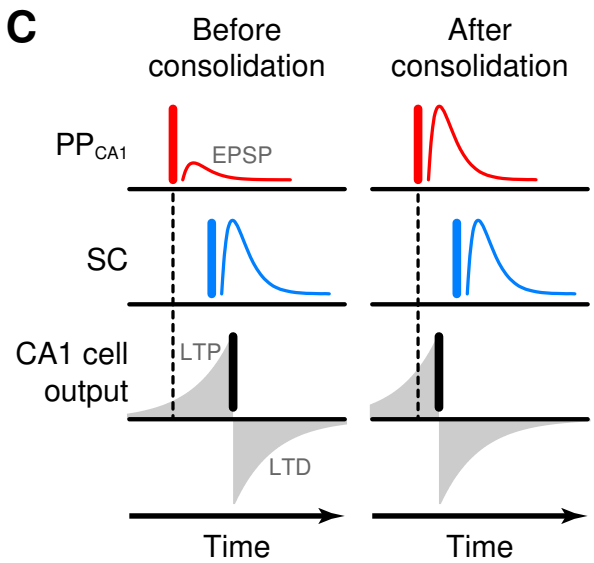

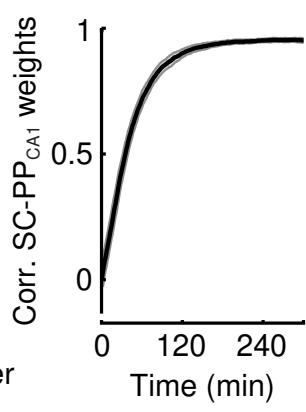

Figure 1. A mechanistic basis for systems memory consolidation. (A) Circuit motif for the parallel pathway theory. Cue-response associations are initially stored in an indirect synaptic pathway (blue) and consolidated into a parallel direct pathway (red). (B) Hippocampal connectivity. The entorhinal cortex projects to CA1 through an indirect pathway via DG-CA3 and the Schaffer collaterals (SC, blue arrow), and through the direct perforant path $\left(\mathrm{PP}_{\mathrm{CA} 1}\right.$, red arrow). (C) Model of consolidation through STDP. Left: before consolidation, a strong SC input (middle, blue vertical bar) causes a large EPSP and triggers a spike in CA1 (bottom, black vertical bar). A weak $\mathrm{PP}_{\mathrm{CA} 1}$ input (top, red) that precedes the SC input is potentiated by STDP. Right: after consolidation through STDP, the $\mathrm{PP}_{\mathrm{CA} 1}$ input (top) can trigger a spike in CA1 by itself (bottom). (D-E) Consolidation in a single integrate-and-fire CA1 cell receiving 1000 $\mathrm{PP}_{\mathrm{CA} 1}$ and $1000 \mathrm{SC}$ excitatory inputs. (D) $\mathrm{PP}_{\mathrm{CA} 1}$ activity consists of independent poisson spike trains; the $\mathrm{SC}$ activity is an exact copy of the $\mathrm{PP}_{\mathrm{CA} 1}$ activity, delayed by $5 \mathrm{~ms}$. (E) Consolidation of a synaptic weight pattern from non-plastic SC synapses to plastic $\mathrm{PP}_{\mathrm{CA} 1}$ synapses. Left and middle: normalized synaptic weights before and after consolidation. Right: time course of correlation between $\mathrm{SC}$ and $\mathrm{PP}_{\mathrm{CA} 1}$ weight vectors during consolidation (mean \pm SEM for 10 trials). 
associated response. The same cue reaches CA1 also through the perforant path, a parallel, direct pathway $\left(\mathrm{PP}_{\mathrm{CA} 1}\right.$, Fig. $1 \mathrm{~B}$, red). We assume that this direct input from EC initially fails to trigger spikes because the synaptic weight pattern in the

$\mathrm{PP}_{\mathrm{CA} 1}$ does not match the cue. However, $\mathrm{PP}_{\mathrm{CA} 1}$ inputs that are activated by the cue precede the spikes in CA1 pyramidal cells that are triggered by the indirect pathway by 5-15 ms (Yeckel and Berger, 1990). Presynaptic spikes preceding postsynaptic spikes with a short delay favour selective long-term potentiation by spike timing-dependent plasticity (STDP, Fig. 1C) (Bi and Poo, 1998; Markram et al., 1997; Gerstner et al., 1996). Consequently, cue-driven PP $\mathrm{CA}_{1}$ synapses onto activated CA1 cells are strengthened until the memory that was initially stored in the indirect pathway can be recalled via the direct pathway alone. The indirect pathway thus acts as a teacher for the direct pathway.

To illustrate this mechanism, we first used a simple integrate-and-fire neuron model of a CA1 cell that receives inputs through the $\mathrm{SC}$ and the PP $\mathrm{CA}_{1}$. We also considered the two pathways to contain the same number of synapses and transmit identical spike patterns apart from a 5-ms delay in the SC (Fig. 1D). Consolidation then corresponds to copying the synaptic weight pattern of the $\mathrm{SC}$ to the $\mathrm{PP}_{\mathrm{CA} 1}$. In line with our hypothesis, such a consolidation was indeed achieved by STDP in the PP $\mathrm{CA1}_{\text {synapses }}$ (Fig. 1E). A consolidation in the opposite direction, i.e., from the $\mathrm{PP}_{\mathrm{CA} 1}$ to the SC cannot be achieved by STDP, because the temporal order of spiking activity is reversed and hence does not favour synaptic potentiation (Supplementary Fig. 1). A mathematical analysis suggests that STDP in the direct pathway implements a linear regression that approximates the input-output mapping of the indirect pathway by that of the direct pathway (Supplementary Sec. 1, 21).

\section{Consolidation of spatial representations}

So far, we illustrated the PPT in a simple integrate-and-fire model, in which the neural representations in the two pathways were identical, leading to a very strong correlation between the inputs to CA1. We next show the robustness of the suggested mechanism in a more biologically plausible setting.

Firstly, we show that the mechanism is robust to differing cue representations in the 
two pathways and to weaker correlations among them (O'Neill et al. 2017$)$. To this 105 end, we used place cell representations (O'Keefe and Dostrovsky, 1971) for the SC input ${ }_{106}$ from CA3 and grid cell representations (Hafting et al., 2005, Moser et al., 2008) for the ${ }_{107}$ $\mathrm{PP}_{\mathrm{CA} 1}$ input from EC (Fig. 2A).

Secondly, we show that the suggested mechanism is compatible with the biophysical properties of CA1 neurons, which receive inputs in different subcellular compartments. To this end, we simulated a multicompartmental CA1 pyramidal cell (Fig. 2B) that was endowed with active ion channels supporting backpropagating action potentials and dendritic calcium spikes (Fig. 2C, Methods).

The use of spatial representations in the input pathways allows us to consider simple forms of memories in a navigational context, in which a given location on a linear track is associated with the activity of a given CA1 cell. Effectively, such an association generates a CA1 place cell. In line with the PPT, we assumed that the spatial selectivity of this CA1 place cell is initially determined solely by the indirect pathway via the SC, i.e., by place cell input from CA3. The goal of systems memory consolidation is then to transfer this spatial association to the direct input, which reaches the CA1 cell via the $\mathrm{PP}_{\mathrm{CA} 1}$ derived from grid cells in EC. In other words, place-cell input should supervise grid-cell input to develop a place-cell tuning.

SC place field inputs were modelled by synapses that were active only in a small region of the track, whereas individual $\mathrm{PP}_{\mathrm{CA} 1}$ grid cell inputs were active in multiple, evenly spaced regions along the track (Fig. 2A). The SC and PP $\mathrm{CA}_{1}$ inputs projected to proximal and distal dendrites, respectively (Fig. 2B, Stuart et al. 2007). Synapses were initialised such that the SC input conductances were spatially tuned and resulted in place field-like activity in the CA1 cell, while the $\mathrm{PP}_{\mathrm{CA} 1}$ input had no spatial tuning (Fig. 2D).

During consolidation, $\mathrm{SC}$ and $\mathrm{PP}_{\mathrm{CA} 1}$ input to the CA1 cell consisted of replays of previously encountered sequences of locations (Skaggs and McNaughton, 1996; Lee and Wilson, 2002), with a replay speed 20 times faster than physical motion Lee and Wilson, 2002). During replay, the SC input led to somatic spikes, which in turn triggered backpropagating action potentials that caused calcium spikes in the distal dendrites where the PP $\mathrm{CA}_{1}$ synapses arrive (Fig. $2 \mathrm{E}$, Larkum et al., 1999). Through synaptic plasticity, $\mathrm{PP}_{\mathrm{CA} 1}$ synapses active in the place field of the neuron were 
A
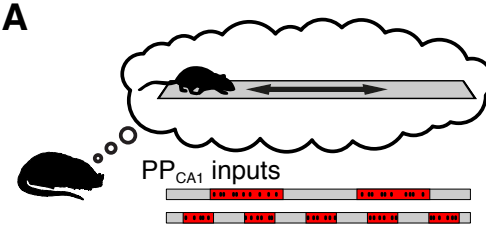

SC inputs

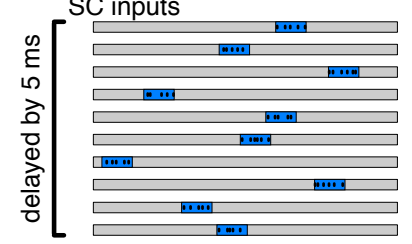

D

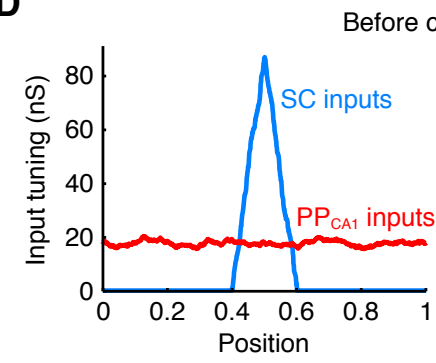

$\mathbf{F}$

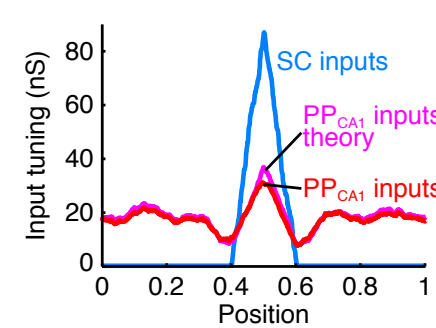

B

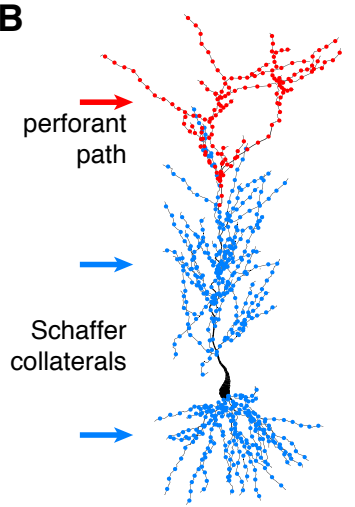

C
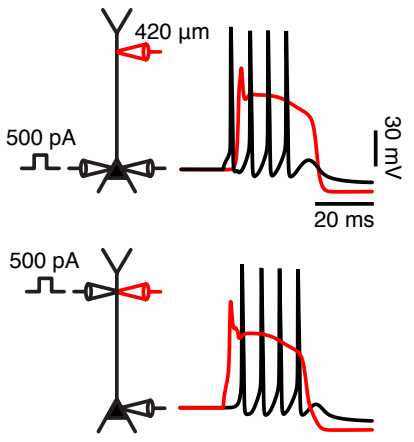

$\mathbf{E}$

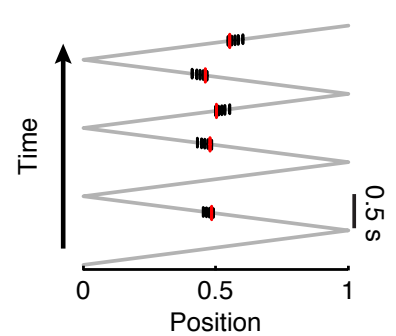

G

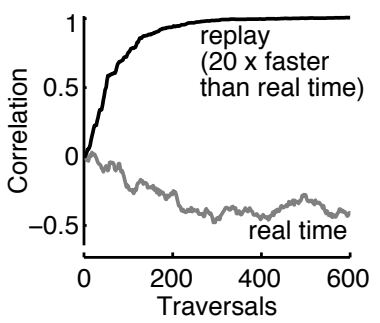

Figure 2. Consolidation of spatial representations. (A) Replay of $\mathrm{PP}_{\mathrm{CA} 1}$ and $\mathrm{SC}$ activity during sleep. $500 \mathrm{PP}_{\mathrm{CA} 1}$ inputs and $2500 \mathrm{SC}$ inputs are spatially tuned on a linear track with periodic grid fields (top, red) and place fields (bottom, blue). Spiking activities are independent Poisson processes (10 spikes/s) inside place/grid fields, otherwise silent. SC activity is delayed by $5 \mathrm{~ms}$. (B) Multi-compartmental model of a reconstructed CA1 pyramidal neuron (see Methods). $\mathrm{PP}_{\mathrm{CA} 1}$ and $\mathrm{SC}$ inputs project to distal apical tuft dendrites (red dots) and proximal apical and basal dendrites (blue dots). (C) Active neuron properties. Top: somatic sodium spike (black) propagates to the distal tuft and initiates a dendritic calcium spike (red) and further sodium spikes. Bottom: dendritic calcium spike leads to bursts of somatic spikes. (D) Spatial tuning before consolidation. SC provides place field-tuned input to the CA1 cell (left, blue), which yields spatially tuned spiking activity (right, blue); $\mathrm{PP}_{\mathrm{CA} 1}$ input is not spatially tuned (left, red), and (alone) triggers low and untuned spiking activity (right, red). (E) Somatic and dendritic activity during consolidation. During replay, SC input generates backpropagating sodium spikes (black vertical lines) that generate dendritic calcium spikes (red). (F) After consolidation. Spatial tuning is consolidated from the indirect $\mathrm{SC}$ pathway into the direct $\mathrm{PP}_{\mathrm{CA} 1}$ pathway. Left: spatial tuning of total $\mathrm{PP}_{\mathrm{CA} 1}$ input (red) approaches theoretically derived $\mathrm{PP}_{\mathrm{CA} 1}$ input tuning (magenta; see Methods). Right: CA1 output is place field-tuned through either $\mathrm{SC}$ or $\mathrm{PP}_{\mathrm{CA} 1}$ input alone. $(\mathrm{G})$ Evolution of correlation between actual and optimal $\mathrm{PP}_{\mathrm{CA} 1}$ input tuning (see $\mathrm{F}$ ) for replay speeds corresponding to hippocampal replay events (black) and real-time physical motion (grey). Position in D, E, and F normalised to [0,1]. 
potentiated. Over time, the $\mathrm{PP}_{\mathrm{CA} 1}$ input adopted the spatial tuning of the $\mathrm{SC}$ input

(Fig. 2F, left) and reproduced the original SC-induced place field output (Fig. 2F, right). ${ }^{138}$

Therefore, the PPT mechanism consolidated associations even though 1) the spatial 139

representations in the two pathways differed (cf. also mathematical analysis in

Supplementary Sec. 2) and 2) the two pathways targeted different neuronal

compartments in the CA1 neuron with complex morphology. Note that consolidation

failed when replay speed was reduced to that of physical motion (Fig. $2 \mathrm{G}$ ), because the

time scale of rate changes in place and grid cell activity is then much longer than the

delay between the two pathways and the time scale of STDP (Supplementary Sec. 3 \&

Supplementary Fig. 2). Accelerated replay during sleep (Lee and Wilson, 2002) hence

supports systems memory consolidation within the PPT by aligning the time scales of neural activity and synaptic plasticity (D'Albis et al. 2015).

\section{Consolidation of place-object associations in multiple}

\section{hippocampal stages}

Ultimately, to consolidate memories into neocortex, they have to move beyond the

$\mathrm{PP}_{\mathrm{CA1}}$. Notably, the $\mathrm{PP}_{\mathrm{CA} 1}$ is itself part of an indirect pathway from EC to the

subiculum (SUB) that is shortcut by a direct connection from EC to SUB (referenced as

$\mathrm{PP}_{\mathrm{SUB}}$; Fig. 3A, left; Amaral (1993)). This suggests that the PPT can be reiterated to further consolidate memories from the $\mathrm{PP}_{\mathrm{CA} 1}$ to the $\mathrm{PP}_{\mathrm{SUB}}$ and beyond.

To illustrate this idea, we considered a standard paradigm for memory research in rodents: the Morris water maze (Morris and Lecar, 1981). In the water maze, the rodent needs to find a submerged platform (object), i.e., it must store an object-place association. Thus this paradigm requires neural representations of objects (such as the submerged platform) and places. We hence constructed a model in which subregions of the hippocampal formation included neurons that encode places and neurons that encoded the identity of objects (Fig. 3A, right).

For simplicity and computational efficiency we switched to a rate-based neuron model Methods. An object was chosen from a set of 128 different objects and placed in a circular open field environment (Fig. $3 \mathrm{~B}$, top). We implemented object-to-place associations in our model by enhancing, as before, synaptic connections in the SC, but 
A

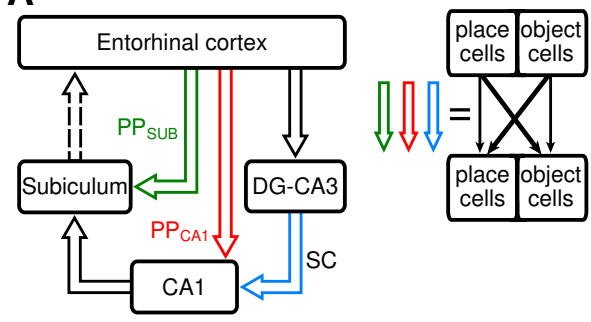

B
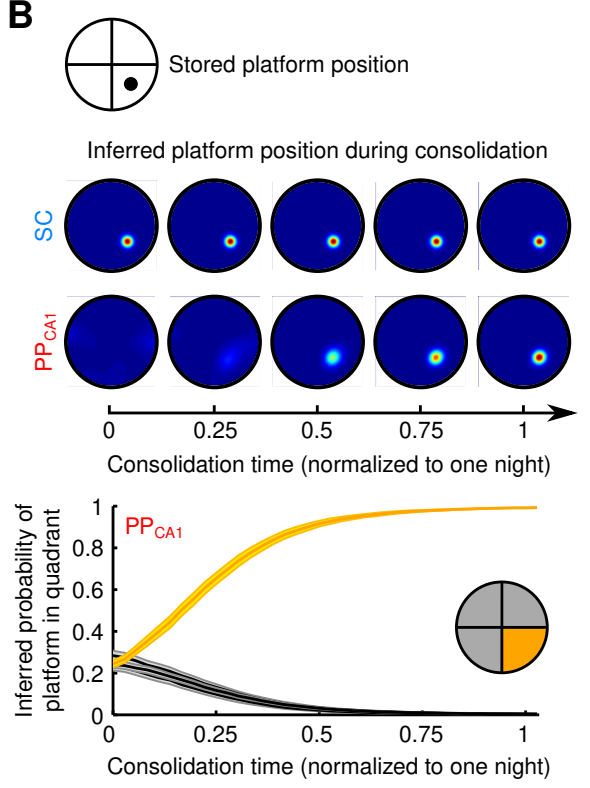

C
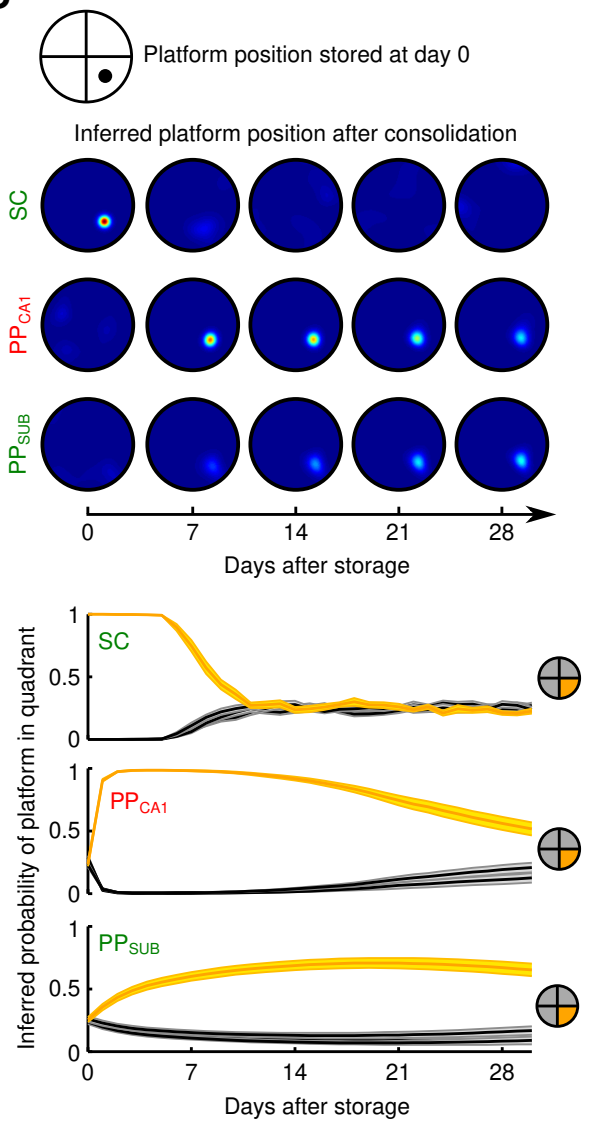

D
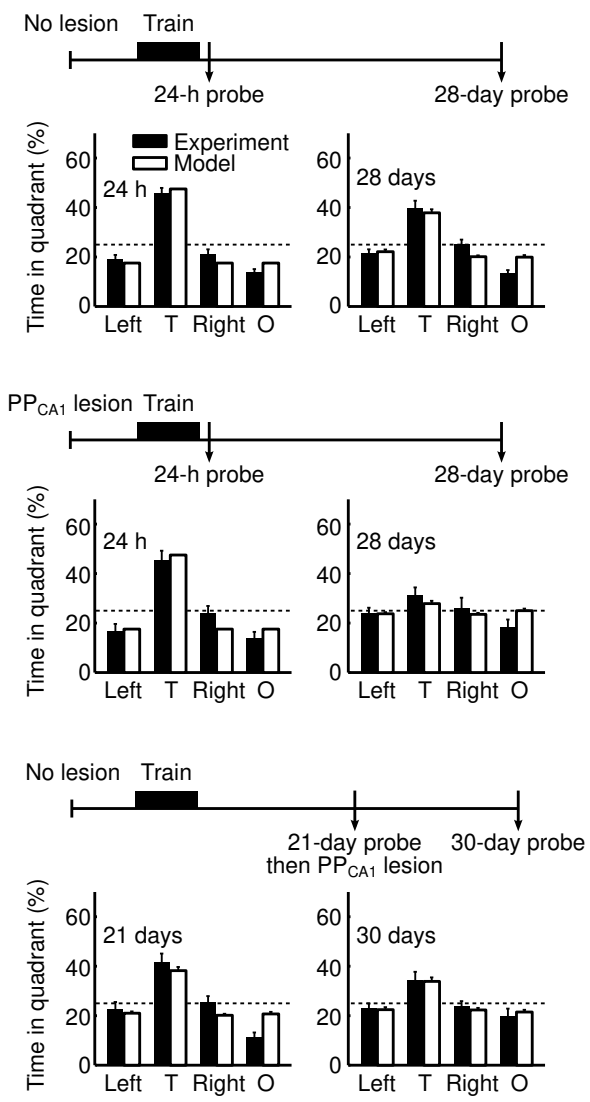

Figure 3. Consolidation of place-object associations in multiple hippocampal stages (A) Structure of the extended model. PP $\mathrm{SUB}_{\mathrm{SU}}$ : perforant path to the subiculum. Each area (EC, DG-CA3, CA1, SUB) contains object-coding and place-coding populations. Open arrows: all-to-all connections between these areas. (B) Decoding of consolidated associations. Top: The location of a platform in a circular environment is stored as an object-place association in the SC (thick diagonal arrows in A, right). Middle: Platform position probability maps given the platform object cue, inferred from the $\mathrm{CA} 1$ output resulting from $\mathrm{SC}$ or $\mathrm{PP}_{\mathrm{CA} 1}$ alone, at different times during consolidation Methods). Bottom: Platform-in-quadrant probabilities ( \pm SEM) given $\mathrm{PP}_{\mathrm{CA} 1}$ input alone during consolidation. Quadrant with correct platform position (target quadrant) in orange. (C) Consolidation from $\mathrm{SC}$ to $\mathrm{PP}_{\mathrm{CA} 1}$ and to $\mathrm{PP}_{\mathrm{SUB}}$ over four weeks. Each day, a new association is first stored in $\mathrm{SC}$ and then partially consolidated. An association on day 0 is monitored in $\mathrm{SC}, \mathrm{PP}_{\mathrm{CA} 1}$, and $\mathrm{PP}_{\mathrm{SUB}}$. Panels as in $\mathrm{B}$. (D) Effects of $\mathrm{PP}_{\mathrm{CA} 1}$ lesions on memory consolidation, model and experiment (data with permission from Remondes and Schuman (2004)). Histograms of time ( \pm SEM) spent in quadrants at different delays after memory acquisition ("probe"). Dashed lines at $25 \%$ are chance levels. T: target quadrant; Left, Right: adjacent quadrants; O: opposite quadrant. Top: Control without lesion. Middle: Lesion before memory acquisition. Bottom: Lesion 21 days after memory acquisition. 
now between object-encoding neurons in CA3 and place-specific neurons in CA1

(Fig. 3A, right). Here, we did not consider place-to-object associations. These are less

relevant for the water maze task, where the task is to recall the location of a given

object - the platform - rather than to recall which object was encountered at a given

location. We tested object-to-place associations stored in the SC by activating the

object representation in EC - as a memory cue - and determining the activities in

CA1, triggered by the SC alone. From these activities we inferred a spatial probability map of the recalled object location (Fig. 3B; Methods).

We first stored a single object-place assocation in the SC. During a subsequent consolidation cycle — representing one night — place and object representations in EC were then randomly and independently activated. Consistent with our previous results, the object-place association was gradually consolidated from the $\mathrm{SC}$ to the $\mathrm{PP}_{\mathrm{CA} 1}$ : after one night of consolidation, the correct spatial probability map of an object location was inferrable from CA1 activity triggered by the $\mathrm{PP}_{\mathrm{CA} 1}$ alone (Fig. $\left.3 \mathrm{~B}\right)$.

To track the consolidation process over longer times, we assumed that a new random object-place association is stored in the SC every day. This caused a decay of previous SC memory traces due to interference with newly stored associations (Fig. $3 \mathrm{C}$, Lux et al. (2016); Fusi and Abbott (2007)). During the night following each day, associations in the $\mathrm{SC}$ were partially consolidated into the $\mathrm{PP}_{\mathrm{CA} 1}$, such that the consolidated association could be decoded from the $\mathrm{PP}_{\mathrm{CA} 1}$ after a single night, but previously consolidated associations were not entirely overwritten. As a result, object-place associations were maintained in the $\mathrm{PP}_{\mathrm{CA} 1}$ for longer periods than in the $\mathrm{SC}$, thus extending their memory lifetime (Fig. $3 \mathrm{C}$ ). Eventually, a given $\mathrm{PP}_{\mathrm{CA} 1}$ memory trace would also degrade as new interfering memories from the SC are consolidated. However, as noted above, the $\mathrm{PP}_{\mathrm{CA} 1}$ itself is part of an indirect pathway from EC to the SUB, for which there is in turn a parallel, direct perforant pathway $\mathrm{PP}_{\mathrm{SUB}}$. The association in the $\mathrm{PP}_{\mathrm{CA} 1}$ (and SC) could therefore, in turn, be partially consolidated into the PP $\mathrm{SUB}_{\mathrm{B}}$, further extending memory lifetime (Fig. 3 3 ).

The model suggests that the $\mathrm{PP}_{\mathrm{CA} 1}$ serves as a transient memory buffer that mediates a further consolidation into additional shortcut pathways downstream. This hypothesis is supported by navigation studies in rats. Using PP $\mathrm{CA1}_{\mathrm{H}}$ lesions, Remondes and Schuman (2004) have shown that the $\mathrm{PP}_{\mathrm{CA} 1}$ is not required for the original 
acquisition of spatial memories, but that it is critically involved in their long-term

maintenance. However, lesioning the $\mathrm{PP}_{\mathrm{CA} 1} 21$ days after acquiring a memory did not

disrupt spatial memories, suggesting that the $\mathrm{PP}_{\mathrm{CA} 1}$ is not the final storage site

(Fig. 3D) and further supporting the idea that the $\mathrm{PP}_{\mathrm{CA} 1}$ is important to enable a

transition from short-term to long-term memories.

To test whether our model could reproduce these experimental results, we simulated $\mathrm{PP}_{\mathrm{CA} 1}$ lesions either before the acquisition of an object-place association or 21 days

later. Assuming that the rat's spatial exploration is determined by the probability map of the object location (Herrnstein, 1961), the model provided predictions for the time spent in different quadrants of the environment, which were in quantitative agreement with the data for all experimental conditions (Fig. 3D). Our model thus suggests that a hierarchical reiteration of parallel shortcuts — the central circuit motif of the PPT could explain these experiments.

\section{Consolidation from hippocampus into neocortex by a hierarchical nesting of consolidation circuits}

Given that shortcut connections are widespread throughout the brain (Van Essen et al. 1992 , Morgenstern et al., 2016, Constantinople and Bruno, 2013), we next hypothesized that a reiteration of the PPT can also achieve systems consolidation from hippocampus into neocortex. To test this hypothesis, we studied a network model (Fig. 4A), in which the hippocampus (now simplified to a single area) receives input from a hierarchy of cortical areas, representing, e.g., a sensory system. It provides output to a different hierarchy of areas, representing, e.g., the motor system. The network also contained shortcut connections that bypassed the hippocampus Methods. As in the previous section, new memories were stored in the hippocampus every day - leading to a decay of previously stored hippocampal memories — and were consolidated by Hebbian plasticity in parallel pathways. Tracing a specific memory over time revealed a gradual fonsolidation into the cortical shortcut connections, forming a "memory wave" Roxin and Fusi, 2013) that travels from hippocampus into neocortex (Fig. 4 B, Supplementary Sec. 4). By exponentially decreasing the shortcut learning rate with distance from the hippocampus, a power-law decay of memories can be observed in the union of all 
shortcuts, e.g., by reading out the shortcut with the strongest memory trace at any moment in time (Fig. $4 \mathrm{~B}$ ). This observation is in line with a rich history of psychological studies on the mathematical shape of forgetting curves (Wixted, 2004).

Notably, we achieved memory retention times of years through only a small number $(\sim 5)$ of iterations of the PPT. Finally, we found that memory retrieval accelerates during consolidation (Fig. 4C), in line with consolidation studies for motor skills (Walker et al. 2002). In our consolidation model, the time to recall decreases because the path from peripheral input to output becomes shorter through the use of more direct (peripheral) shortcut connections (Fig. 4 A and B).

A

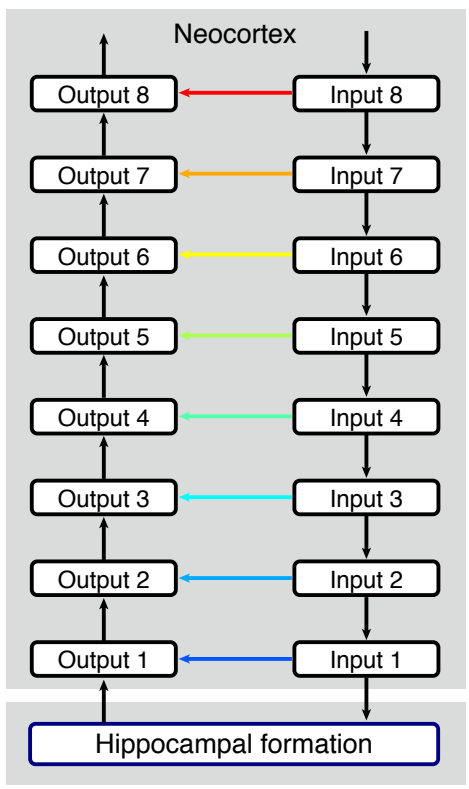

B

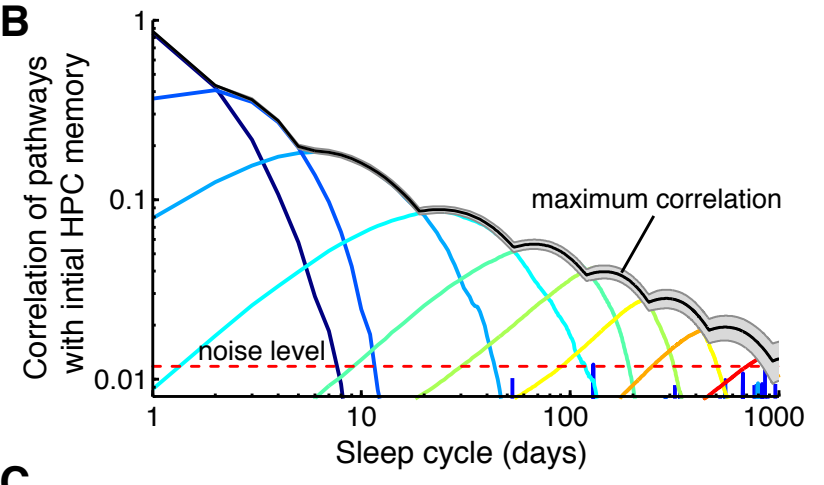

C

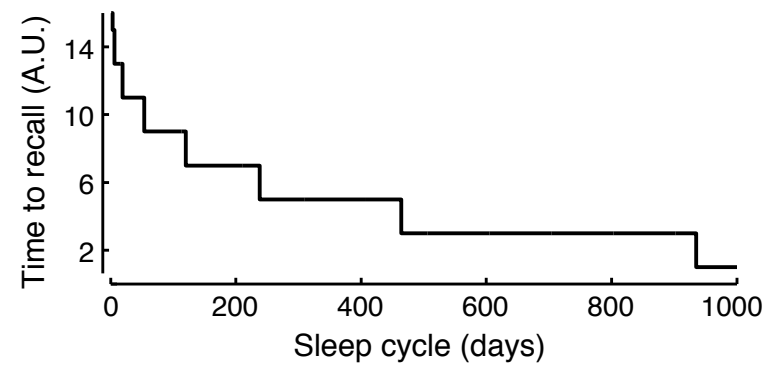

Figure 4. Consolidation from hippocampus into neocortex by a hierarchical nesting of consolidation circuits. (A) Schematic of the hierarchical model. The hippocampal formation (HPC) is connected to cortical input circuit 1 and output circuit 1. Increasing numbers indicate circuits further from the HPC and closer to the sensory/motor periphery. Each direct connection at one level (e.g., dark blue arrow between input 1 and output 1) is part of the indirect pathway of the next level (e.g., for pathways from input 2 to output 2). Learning rates of the direct connections decrease exponentially with increasing level (i.e., from blue to red). (B) Memories gradually propagate to circuits more distant from the HPC. The correlation of the initial HPC weights with the direct pathways is shown as a function of time and reveals a memory wave from HPC into neocortex. The maximum of the output circuits follows approximately a power-law (black curve). Noise level indicates chance level correlations between pathways. (C) Consolidated memories yield faster responses (from sensory periphery, e.g., Input 8, to system output) because these memories are stored in increasingly shorter synaptic pathways. 


\section{Discussion}

We proposed the parallel pathway theory (PPT) as a mechanistic basis for systems memory consolidation. This theory relies on two abundant features in the nervous system: parallel shortcut connections between brain areas and Hebbian plasticity. We applied the PPT to hippocampus-dependent memories and showed that the proposed mechanism can transfer memory associations across parallel synaptic pathways. This transfer is robust to different representations in those pathways and requires only weak correlations. Our theory is in quantitative agreement with lesion studies of the perforant path in rodents (Remondes and Schuman, 2004) and is able to reproduce forgetting curves that follow a power-law as observed in humans (Wixted, 2004).

\section{Theory requirements}

In addition to the anatomical motif of shortcut connections and Hebbian synaptic plasticity, the parallel pathway theory relies on four further requirements during the consolidation phase.

First, temporal correlations between the inputs from the two input pathways are necessary. For example, in the hippocampus the mechanism assumes such correlations between $\mathrm{CA} 3$ and $\mathrm{EC}$ inputs to CA1 during consolidation, the presence of which is not fully resolved (O'Neill et al., 2017). A consolidation from hippocampus into neocortex would require correlations between cortical and hippocampal activity (Ji and Wilson, 2007).

Second, the direct pathway should be plastic during consolidation, while the stored associations in the indirect path remain sufficiently stable. In practise, this requires the degree of plasticity to differ between periods of storage and consolidation (e.g., due to neuromodulation (Hasselmo, 1999, Papouin et al., 2017)), in a potentially

pathway-dependent manner. In other words, the requirement is that the content of a memory should not be altered much while creating a backup.

Third, plasticity in the shortcut pathway should be driven by a teaching signal from the indirect pathway. This can be achieved by STDP in combination with longer transmission delays in the indirect pathway, as suggested here, but other neural implementations of supervised learning may be equally suitable Legenstein et al. 2005. 
Pfister et al. 2006, Urbanczik and Senn, 2014).

Fourth, within the present theory, a systematic decrease in learning rates within the consolidation hierarchy (Fig. 4) is needed to achieve memory lifetimes on the order of years. That is, synapses involved in later stages of consolidation should be less plastic during consolidation periods such as sleep, as also suggested by Roxin and Fusi (2013). A similar approach for obtaining power-law forgetting has been used in models of synaptic memory consolidation (Fusi et al. 2005, Benna and Fusi, 2016).

\section{What limits systems memory consolidation?}

Our account of systems memory consolidation explains how memories are re-organized and transferred across brain regions. However, certain forms of episodic memory remain hippocampus-dependent throughout life (Winocur and Moscovitch, 2011).

In the context of the present model, this restriction could result from different factors. The PPT simplifies memory engrams by replacing multisynaptic by monosynaptic connections whenever possible. However, a shortcut pathway may not be present anatomically, or it may not host an appropriate representation for a given cue-response association in question. For example, it may be difficult to consolidate a complex visual object detection task into a shortcut from primary visual cortex (V1) to a decision area, because the low-level representation of the visual cue in V1 may not allow it (DiCarlo and Cox, 2007, Majaj et al. 2015). The same applies to tasks that require a mixed selectivity of neural responses (Rigotti et al. 2013). Such tasks cannot be fully consolidated into shortcuts with simpler representations of cues and/or responses that do not allow a linear separation of the associations. On the basis of similar arguments, early work suggested that the hippocampus could be critical for learning tasks that are not linearly separable (Sutherland and Rudy, 1989).

Within the present framework, the consolidated memory is in essence a linear approximation of the original cue-response association (Supplementary Sec. 2). The resulting simplification of the memory content could underlie the commonly observed semantisation of memories and the loss of episodic detail (Winocur et al. 2010 , Winocur and Moscovitch, 2011). Such a semantisation could already occur in the earliest shortcut connections (Schapiro et al. 2017), but could also gradually progress in 


\section{Relation to phenomenological models of systems consolidation}

The basic mechanism of our theory explains memory transfer between brain regions, interference of new memory items with older memories (McCloskey and Cohen, 1989).

In our model, later - presumably neocortical — shortcut connections have lower learning rates to achieve longer memory retention times. Interleaved learning could be achieved by interleaved replay (Foster, 2017; Schuck and Niv, 2019, Liu et al., 2019) during consolidation. Thereby, the results of CLST can be directly applied to learning in shortcuts in our model, such as the rapid neocortical consolidation of new memories that are in line with a previously learned schema (Tse et al., 2007, 2011, McClelland, 2013).

Limitations of memory transfer between brain regions - as discussed above - can impair the consolidation process, resulting in memories that remain hippocampusdependent throughout life. Hence, our theory is also in agreement with the Multiple Trace Theory (MTT) (Nadel and Moscovitch, 1997) and the Trace Transformation Theory (TTT) (Winocur et al. 2010, Winocur and Moscovitch, 2011).

The MTT postulates that memories are re-encoded in the hippocampus during retrieval, generating multiple traces for the same memory. Our model maintains multiple memory traces in different shortcut pathways, even without a retrieval-based re-encoding. The consolidation mechanism of the PPT, however, could also transfer a specific memory multiple times if it is re-encoded during retrieval. If neocortex extracts statistical regularities from a collection of memories (McClelland et al. 1995$)$, the consolidation of such a repeatedly re-encoded memory could then lead to a gist-like, more semantic version of that memory in neocortex (Nadel and Moscovitch, 1997, Winocur and Moscovitch, 2011; Levine et al., 2002), as emphasised by the TTT.

The premise of our model is that memories are actively transferred between brain regions. This premise has recently been subject to debate Yonelinas et al. 2019a; 
Antony and Schapiro, 2019, Yonelinas et al., 2019b), following the suggestion of the

Contextual Binding (CB) theory. The CB theory argues that amnesia in lesion studies

and replay-like activity can be explained by simultaneous learning in hippocampus and neocortex, together with interference of contextually similar episodic memories (Yonelinas et al. 2019a). Note, however, that our theory does not exclude a simultaneous encoding in neocortex and hippocampus, which can be combined with active consolidation (Dudai et al., 2015, Pöhlchen and Schönauer, 2020).

Hence, our mechanistic approach is in agreement with and may allow for a unification of several phenomenological theories of systems consolidation.

\section{Consolidation of non-declarative memories}

Given that shortcut connections are widespread throughout the central nervous system (Van Essen et al., 1992, Morgenstern et al., 2016), the suggested mechanism may also be applicable to the consolidation of non-declarative memories, e.g., of perceptual (Karni et al., 1994) and motor skills (Brashers-Krug et al., 1996), fear memory Kitamura et al. 2017) or to the transition of goal-directed to habitual behaviour Aarts and Dijksterhuis, 2000).

Several studies have suggested two-pathway models in the context of motor learning Makino et al. 2016, Pyle and Rosenbaum, 2019, Teşileanu et al., 2017, Murray and Escola, 2020). In particular, Murray and Escola (2020) recently used a two-pathway model to investigate how repeated practice affects future performance and leads to habitual behaviour. While their model does not incorporate an active consolidation mechanism or multiple learning stages, the basic mechanism is the same: A fast learning pathway from cortex to sensorimotor striatum first learns a motor skill and then teaches a slowly learning pathway from thalamus to striatum during subsequent repetition.

\section{Limitations of the model and future directions}

The present work focuses on feedforward networks and local learning rules. Hence, the model cannot address how systems memory consolidation affects the representation of sensory stimuli and forms schemata that facilitate future learning Tse et al. 2007. 2011) because representation learning typically requires a means of backpropagating 
information through the system, e.g., by feedback connections (Lillicrap et al., 2020).

The interaction of synaptic plasticity with recurrent feedback connections generates a

high level of dynamical complexity, which is beyond the scope of the present study. Our

On the mechanistic level, the theory predicts temporally specific deficits in memory consolidation when relevant shortcut connections are lesioned, that is, a tight link between the anatomical organisation of synaptic pathways and their function for memory. These predictions may be most easily tested in non-mammalian systems, where connectomic data are available (Xu et al. 2020).

The parallel pathway theory could provide an inroad to a mechanistic understanding of the transformation of episodic memories into more semantic representations. This could be modelled, e.g, by encoding a collection of episodic memories that share statistical regularities and studying the dynamics of statistical learning and semantisation in the shortcut connections during consolidation. Such future work may allow to ultimately bridge the gap between memory consolidation on the mechanistic level of synaptic computations and the behavioural level of cognitive function. 


\section{Acknowledgements}

We would like to thank Naomi Auer, Tiziano D'Albis, and Robert Gütig for discussions and feedback on the manuscript.

This work was funded by the German Research Foundation (DFG, Project number

327654276 - SFB 1315 to HS, SS, and RK), the German Federal Ministry of Education

379 and Research (Bernstein Award, FKZ GQ1201 to HS; 01GQ1705 to RK; 01 GQ0901 and 01GQ1403 to SS), and the Einstein Foundation Berlin (to MR and SS).

\section{Author contributions}

M.R, U.B, S.S, H.S. and R.K. conceived the study. M.R. and U.B. conducted the

simulations. H.S. performed the mathematical analysis. D.A. reproduced part of the results independently. All authors wrote the manuscript.

\section{Competing Interests statement}

We declare that none of the authors have competing financial or non-financial interests. $\quad 387$ 


\section{References}

Aarts, H., and A. Dijksterhuis, Habits as knowledge structures: Automaticity in goal-directed behavior, Journal of Personality and Social Psychology, 78(1), 53-63, 2000.

Abraham, W. C., and A. Robins, Memory retention - the synaptic stability versus plasticity dilemma, Trends in Neurosciences, 28(2), 73-78, 2005.

Amaral, D. G., Emerging principles of intrinsic hippocampal organization, Current Opinion in Neurobiology, 3(2), 225-229, 1993.

Antony, J. W., and A. C. Schapiro, Active and effective replay: systems consolidation reconsidered again, Nature Reviews Neuroscience, 20(8), 506-507, 2019.

Benna, M. K., and S. Fusi, Computational principles of synaptic memory consolidation, 398 Nature Neuroscience, 19(12), 1697-1706, 2016.

Bi, G.-q., and M.-m. Poo, Synaptic modifications in cultured hippocampal neurons: dependence on spike timing, synaptic strength, and postsynaptic cell type, Journal of Neuroscience, 18, 10,464-10,472, 1998.

Brashers-Krug, T., R. Shadmehr, and E. Bizzi, Consolidation in human motor memory, Nature, 382(6588), 252-255, 1996.

Brodt, S., S. Gais, J. Beck, M. Erb, K. Scheffler, and M. Schönauer, Fast track to the neocortex: A memory engram in the posterior parietal cortex, Science, 362(6418), 1045-1048, 2018.

Constantinople, C. M., and R. M. Bruno, Deep cortical layers are activated directly by thalamus, Science, 340(6140), 1591-1594, 2013.

Debiec, J., J. E. LeDoux, and K. Nader, Cellular and systems reconsolidation in the hippocampus, Neuron, 36(3), 527-538, 2002.

DiCarlo, J. J., and D. Cox, Untangling invariant object recognition, Trends in Cognitive Sciences, 11(8), 333-341, 2007.

Diekelmann, S., and J. Born, The memory function of sleep, Nature Reviews 403 
Dudai, Y., The restless engram: consolidations never end, Annual Review of

Dudai, Y., A. Karni, and J. Born, The consolidation and transformation of memory, Neuron, 88(1), 20-32, 2015.

D’Albis, T., J. Jaramillo, H. Sprekeler, and R. Kempter, Inheritance of hippocampal place fields through Hebbian learning: Effects of theta modulation and phase precession on structure formation, Neural Computation, 27(8), 1624-1672, 2015.

Foster, D. J., Replay comes of age, Annual Review of Neuroscience, 40(1), 581-602, 2017.

Fusi, S., and L. Abbott, Limits on the memory storage capacity of bounded synapses, Nature Neuroscience, 10(4), 485-493, 2007.

Fusi, S., P. Drew, and L. Abbott, Cascade models of synaptically stored memories, Neuron, 45(4), 599-611, 2005.

Gerstner, W., R. Kempter, J. van Hemmen, and H. Wagner, A neuronal learning rule for sub-millisecond temporal coding, Nature, 383(6595), 76-78, 1996.

Grossberg, S., The Adaptive Brain I, Elsevier Science, Amsterdam, 1987.

Hafting, T., M. Fyhn, S. Molden, M. Moser, and E. I. Moser, Microstructure of a spatial map in the entorhinal cortex, Nature, 436(7052), 801-806, 2005.

Hasselmo, M. E., Neuromodulation: acetylcholine and memory consolidation, Trends in Cognitive Sciences, 3(9), 351-359, 1999.

Herrnstein, R. J., Relative and absolute strength of response as a function of frequency of reinforcement, Journal of the Experimental Analysis of Behavior, 4(3), 267-272, 1961.

Ji, D., and M. A. Wilson, Coordinated memory replay in the visual cortex and hippocampus during sleep, Nature Neuroscience, 10(1), 100-107, 2007.

Karni, A., D. Tanne, B. S. Rubenstein, J. J. Askenasy, and D. Sagi, Dependence on REM sleep of overnight improvement of a perceptual skill, Science, 265(5172), 679-682, 1994. 
Kitamura, T., S. K. Ogawa, D. S. Roy, T. Okuyama, M. D. Morrissey, L. M. Smith,

R. L. Redondo, and S. Tonegawa, Engrams and circuits crucial for systems consolidation of a memory, Science, 356(6333), 73-78, 2017.

Kumaran, D., D. Hassabis, and J. L. McClelland, What learning systems do intelligent agents need? Complementary learning systems theory updated, Trends in Cognitive Sciences, 20(7), 512-534, 2016.

Larkum, M. E., J. J. Zhu, and B. Sakmann, A new cellular mechanism for coupling inputs arriving at different cortical layers, Nature, 398(6725), 338-341, 1999.

Lee, A., and M. Wilson, Memory of sequential experience in the hippocampus during slow wave sleep, Neuron, 36(6), 1183-1194, 2002.

Legenstein, R., C. Naeger, and W. Maass, What can a neuron learn with spike-timing dependent plasticity, Neural Computation, 17(11), 2337-2382, 2005.

Leibold, C., and R. Kempter, Sparseness constrains the prolongation of memory lifetime via synaptic metaplasticity, Cerebral Cortex, 18(1), 67-77, 2008.

Levine, B., E. Svoboda, J. F. Hay, G. Winocur, and M. Moscovitch, Aging and autobiographical memory: dissociating episodic from semantic retrieval., Psychology and Aging, 17(4), 677-89, 2002.

Lillicrap, T. P., A. Santoro, L. Marris, C. J. Akerman, and G. Hinton, Backpropagation and the brain, Nature Reviews Neuroscience, 21(6), 335-346, 2020.

Liu, Y., R. J. Dolan, Z. Kurth-Nelson, and T. E. J. Behrens, Human replay spontaneously reorganizes experience, Cell, 178(3), 640-652, 2019.

Lux, V., E. Atucha, T. Kitsukawa, and M. M. Sauvage, Imaging a memory trace over half a life-time in the medial temporal lobe reveals a time-limited role of CA3 neurons in retrieval, eLife, 5, e11,862, 2016.

Majaj, N. J., H. Hong, E. A. Solomon, and J. J. DiCarlo, Simple learned weighted sums of inferior temporal neuronal firing rates accurately predict human core object recognition performance, Journal of Neuroscience, 35(39), 13,402-13,418, 2015. 
Makino, H., E. J. Hwang, N. G. Hedrick, and T. Komiyama, Circuit mechanisms of sensorimotor learning, Neuron, 92(4), 705-721, 2016.

Malenka, R. C., and M. F. Bear, LTP and LTD: An embarassment of riches, Neuron, $44(1), 5-21,2004$.

Markram, H., J. Lübke, M. Frotscher, and B. Sakmann, Regulation of synaptic efficacy by coincidence of postysnaptic APs and EPSPs, Science, 275(5297), 213-215, 1997.

Marr, D., A theory of cerebellar cortex, The Journal of Physiology, 202(2), 437-470, 1969.

McClelland, J. L., Incorporating rapid neocortical learning of new schema-consistent information into complementary learning systems theory, Journal of Experimental Psychology: General, 142(4), 1190-1210, 2013.

McClelland, J. L., B. L. O’Reilly, and R. C. McNaughton, Why there are complementary learning systems in the hippocampus and neocortex: Insights from the successes and failures of connectionist models of learning and memory, Psychological Review, 102(3), 419-457, 1995.

McCloskey, M., and N. J. Cohen, Catastrophic interference in connectionist networks: The sequential learning problem, pp. 109-165, 1989.

Morgenstern, N. A., J. Bourg, and L. Petreanu, Multilaminar networks of cortical neurons integrate common inputs from sensory thalamus, Nature Neuroscience, 19(8), 1034-1040, 2016.

Morris, C., and H. Lecar, Voltage oscillations in the barnacle giant muscle fiber, Biophysical Journal, 35(1), 193-213, 1981.

Moser, E. I., E. Kropff, and M.-B. Moser, Place cells, grid cells, and the brain's spatial representation system, Annual Review of Neuroscience, 31, 69-89, 2008.

Murray, J. M., and G. S. Escola, Remembrance of things practiced: Fast and slow learning in cortical and subcortical pathways, bioRxiv, p. 797548, doi:10.1101/797548, 2020. 
Nadel, L., and M. Moscovitch, Memory consolidation, retrograde amnesia and the hippocampal complex, Current Opinion in Neurobiology, 7(2), 217-227, 1997.

Nakazawa, K., L. D. Sun, M. C. Quirk, L. Rondi-Reig, M. A. Wilson, and S. Tonegawa, Hippocampal CA3 NMDA receptors are crucial for memory acquisition of one-time experience, Neuron, 38(2), 305-315, 2003.

O’Keefe, J., and J. Dostrovsky, The hippocampus as a spatial map: Preliminary evidence from unit activity in the freely-moving rat, Brain Research, 34(1), 171-175, 1971.

O’Neill, J., C. Boccara, F. Stella, P. Schoenenberger, and J. Csicsvari, Superficial layers of the medial entorhinal cortex replay independently of the hippocampus, Science, $355(6321), 184-188,2017$.

Papouin, T., J. M. Dunphy, M. Tolman, K. T. Dineley, and P. G. Haydon, Septal cholinergic neuromodulation tunes the astrocyte-dependent gating of hippocampal NMDA receptors to wakefulness, Neuron, 94(4), 840-854, 2017.

Pyle, R., and R. Rosenbaum, A reservoir computing model of reward-modulated motor learning and automaticity, Neural Computation, 31(7), 1430-1461, 2019.

Pöhlchen, D., and M. Schönauer, Sleep-dependent memory consolidation in the light of rapid neocortical plasticity, Current Opinion in Behavioral Sciences, 33, 118-125, 2020.

Remondes, M., and E. Schuman, Role for a cortical input to hippocampal area CA1 in the consolidation of a long-term memory, Nature, 431(7009), 699-703, 2004.

Rigotti, M., O. Barak, M. R. Warden, X.-J. Wang, N. D. Daw, E. K. Miller, and S. Fusi, ${ }_{522}$ The importance of mixed selectivity in complex cognitive tasks, Nature, 497(7451), ${ }_{523}$ 585-590, 2013. 
Roxin, A., and S. Fusi, Efficient partitioning of memory systems and its importance for memory consolidation, PLoS Computational Biology, 9(7), e1003,146, 2013.

Schapiro, A. C., N. B. Turk-Browne, M. M. Botvinick, and K. A. Norman, Complementary learning systems within the hippocampus: A neural network modelling approach to reconciling episodic memory with statistical learning, Philosophical Transactions of the Royal Society B: Biological Sciences, 372(1711), 20160,049, 2017.

Schuck, N. W., and Y. Niv, Sequential replay of nonspatial task states in the human hippocampus, Science, 364(6447), eaaw5181, 2019.

Sekeres, M. J., M. Moscovitch, and G. Winocur, Mechanisms of memory consolidation and transformation, in Cognitive Neuroscience of Memory Consolidation, edited by N. Axmacher and B. Rasch, pp. 17-44, Springer International Publishing, Switzerland, 2017.

Skaggs, W. E., and B. L. McNaughton, Replay of neuronal firing sequences in rat hippocampus during sleep following spatial experience, Science, 271 (5257), 1870-1873, 1996.

Squire, L. R., and P. Alvarez, Retrograde amnesia and memory consolidation: a neurobiological perspective, Current Opinion in Neurobiology, 5(2), 169-177, 1995.

Squire, L. R., L. Genzel, J. T. Wixted, and R. G. Morris, Memory consolidation, Cold Spring Harbor Perspectives in Biology, 7(8), a021,766, 2015.

Stuart, G., N. Spruston, and M. Häusser, Dendrites, Oxford University Press, Oxford, 2007.

Sutherland, R. J., and J. W. Rudy, Configural association theory: The role of the hippocampal formation in learning, memory, and amnesia, Psychobiology, 17(2), $129-144,1989$.

Teşileanu, T., B. Ölveczky, and V. Balasubramanian, Rules and mechanisms for efficient two-stage learning in neural circuits, eLife, 6, e20,944, 2017. 
Treves, A., and E. T. Rolls, A computational analysis of the role of the hippocampus in $\quad 552$ learning and memory, Hippocampus, 4(3), 373-391, 1994.

Tse, D., R. F. Langston, M. Kakeyama, I. Bethus, P. A. Spooner, E. R. Wood, M. P.

Witter, and R. G. Morris, Schemas and memory consolidation, Science, 316(5821), $76-82,2007$.

Tse, D., T. Takeuchi, M. Kakeyama, Y. Kajii, H. Okuno, C. Tohyama, H. Bito, and

R. G. M. Morris, Schema-dependent gene activation and memory encoding in neocortex, Science, 333(6044), 891-5, 2011.

Urbanczik, R., and W. Senn, Learning by the dendritic prediction of somatic spiking, Neuron, $81(3), 521-528,2014$.

Van Essen, D. C., C. H. Anderson, and D. J. Felleman, Information processing in the primate visual system: an integrated systems perspective, Science, 255(5043), 419-423, 1992.

Walker, M. P., T. Brakefield, A. Morgan, J. A. Hobson, and R. Stickgold, Practice with sleep makes perfect: Sleep-dependent motor skill learning, Neuron, 35(1), 205-211, 2002.

Winocur, G., and M. Moscovitch, Memory transformation and systems consolidation, Journal of the International Neuropsychological Society, 17(5), 766-780, 2011.

Winocur, G., M. Moscovitch, and B. Bontempi, Memory formation and long-term retention in humans and animals: Convergence towards a transformation account of hippocampal-neocortical interactions, Neuropsychologia, 48(8), 2339-2356, 2010.

Wixted, J. T., The psychology and neuroscience of forgetting, Annual Review of Psychology, 55, 235-269, 2004.

$\mathrm{Xu}$, C. S., et al., A connectome of the adult Drosophila central brain, bioRxiv, p. 911859, doi:10.1101/2020.01.21.911859, 2020.

Yeckel, M. F., and T. W. Berger, Feedforward excitation of the hippocampus by afferents from the entorhinal cortex: redefinition of the role of the trisynaptic pathway, Proceedings of the National Academy of Sciences USA, 87(15), 5832-5836, 1990. 
Yonelinas, A. P., C. Ranganath, A. D. Ekstrom, and B. J. Wiltgen, A contextual 


\section{Methods}

\section{Consolidation in a single integrate-and-fire neuron}

For the results shown in Fig. $1 \mathrm{E}$ and Supplementary Fig. 1 we used a single integrate-and-fire model neuron that received excitatory synaptic input. The membrane potential $V(t)$ evolved according to

$$
\tau_{\mathrm{m}} \frac{\mathrm{d} V}{\mathrm{~d} t}=V_{\text {rest }}-V+g_{\mathrm{syn}}(t)\left(E_{\mathrm{syn}}-V\right)
$$

with membrane time constant $\tau_{\mathrm{m}}=20 \mathrm{~ms}$, resting potential $V_{\text {rest }}=-70 \mathrm{mV}$, and synaptic reversal potential $E_{\text {syn }}=0 \mathrm{mV}$. When the membrane potential reached the threshold $V_{\text {thresh }}=-54 \mathrm{mV}$, the cell produced a spike and the voltage was reset to $-60 \mathrm{mV}$ during an absolute refractory period of $1.75 \mathrm{~ms}$.

The total synaptic conductance $g_{\text {syn }}(t)$ in Eq. (1) is denoted in units of the leak conductance and thus dimensionless (parameters are taken from Troyer and Miller (1997)). The total synaptic conductance was determined by the sum of 1000 Schaffer collateral (SC) inputs and 1000 perforant path $\left(\mathrm{PP}_{\mathrm{CA} 1}\right)$ inputs. Activation of input $i$ (where $i$ denotes synapse number) leads to a jump $g_{i}>0$ in the synaptic conductance:

$$
g_{\mathrm{syn}}(t) \rightarrow g_{\mathrm{syn}}(t)+g_{i}
$$

All synaptic conductances decay exponentially,

$$
\tau_{\mathrm{syn}} \frac{\mathrm{d} g_{\mathrm{syn}}}{\mathrm{d} t}=-g_{\mathrm{syn}}
$$

with synaptic time constant $\tau_{\mathrm{syn}}=5 \mathrm{~ms}$. The $\mathrm{PP}_{\mathrm{CA} 1}$ inputs were activated by mutually independent Poisson processes with a mean rate of 10 spikes/s. The activity patterns of the SC fibers were identical to those of the $\mathrm{PP}_{\mathrm{CA} 1}$ fibers but were delayed by $5 \mathrm{~ms}$.

The synaptic peak conductances or weights, $g_{i}$, were either set to a fixed value or were determined by additive STDP (Song et al. 2000). A single pair of a presynaptic spike (at time $t_{\text {pre }}$ ) and a postsynaptic spike (at time $t_{\text {post }}$ ) with time difference 
$\Delta t \equiv t_{\text {pre }}-t_{\text {post }}$ induced a modification of the synaptic weight $\Delta g_{i}$ according to

$$
\Delta g_{i}=L(\Delta t)= \begin{cases}+A^{+} \exp \left(\Delta t / \tau_{\mathrm{STDP}}\right) & \text { if } \Delta t<0 \\ -A^{-} \exp \left(-\Delta t / \tau_{\mathrm{STDP}}\right) & \text { if } \Delta t \geq 0\end{cases}
$$

with $\tau_{\text {STDP }}=20 \mathrm{~ms} . L(\Delta t)$ is the learning window of STDP (Song et al. 2000$)$. Hard upper and lower bounds were imposed on the synaptic weights, such that $0 \leq g_{i} \leq \bar{g}_{\max }$ for all $i$, where the dimensionless maximum synaptic weight was $\bar{g}_{\max }=0.006$.

Parameters $A^{+}=0.005 \cdot \bar{g}_{\max }$ and $A^{-}=1.05 \cdot A^{+}$determine the maximum amounts of LTP and LTD, respectively.

Synaptic weights were initialized to form a bimodal distribution, such that it agrees with the steady state weight distribution resulting from additive STDP, when presynaptic input consists of uncorrelated Poisson spike trains (Song et al. 2000). Specifically, half the weights were sampled from an exponential distribution with mean $0.05 \cdot \bar{g}_{\max }$, the other half as $\bar{g}_{\max }$ minus that same exponential distribution.

The dynamics were integrated numerically using the forward Euler method, with an integration time step of $0.1 \mathrm{~ms}$.

\section{Consolidation of spatial representations in a multi-compartment neuron model}

The results presented in Fig. 22C-G relied on numerical simulations of a conductance-based compartmental model of a reconstructed CA1 pyramidal cell (cell n128 from Cannon et al. (1998)). Passive cell properties were defined by the membrane

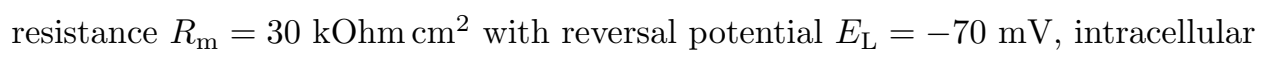
resistivity $R_{\mathrm{i}}=150 \Omega \mathrm{cm}$, and membrane capacitance $C_{\mathrm{m}}=0.75 \mu \mathrm{F} / \mathrm{cm}^{2}$. Dendrites were discretized into compartments with length smaller than 0.1 times the frequency-dependent passive space constant at $100 \mathrm{~Hz}$. Three types of voltage-dependent currents and one calcium-dependent current, all from Mainen and Sejnowski 1996), were distributed over the soma and dendrites. Gating dynamics of the currents evolved according to standard first-order ordinary differential equations. The steady state (in)activation functions $x_{\infty}$ and voltage-dependent time constants $\tau_{\infty}$ for each gating variable (i.e., $x=m, h, n$; see below) were calculated from a first-order 
reaction scheme with forward rate $\alpha_{x}$ and backward rate $\beta_{x}$ according to

$x_{\infty}(V)=\alpha_{x}(V) /\left(\alpha_{x}(V)+\beta_{x}(V)\right)$ and $\tau_{\mathbf{x}}(V)=1 /\left(\alpha_{x}(V)+\beta_{x}(V)\right)$ where $V$ was the membrane potential. All used current densities and time constants were selected for a temperature of $37^{\circ} \mathrm{C}$ (see Mainen and Sejnowski (1996)).

A fast sodium current, $I_{\mathrm{Na}}$, was distributed throughout the soma $\left(\bar{g}_{\mathrm{Na}}=130 \mathrm{pS} / \mu \mathrm{m}^{2}\right)$ and dendrites $\left(\bar{g}_{\mathrm{Na}}=260 \mathrm{pS} / \mu \mathrm{m}^{2}\right)$, except from the distal apical dendritic tuft,

$$
I_{\mathrm{Na}}=\bar{g}_{\mathrm{Na}} m^{3} h\left(V-E_{\mathrm{Na}}\right),
$$

with reversal potential $E_{\mathrm{Na}}=60 \mathrm{mV}$. The dynamics of activation gating variable $m$ and inactivation gating variable $h$ were characterised by

$$
\begin{aligned}
\alpha_{\mathrm{m}} & =-0.584 \frac{V+30}{\mathrm{e}^{-(V+30) / 9}-1} \\
\beta_{\mathrm{m}} & =0.398 \frac{V+30}{\mathrm{e}^{(V+30) / 9}-1} \\
\alpha_{\mathrm{h}} & =-0.077 \frac{V+45}{\mathrm{e}^{-(V+45) / 5}-1} \\
\beta_{\mathrm{h}} & =0.0292 \frac{V+70}{\mathrm{e}^{(V+70) / 5}-1} .
\end{aligned}
$$

Here and in the following, we dropped units for simplicity, assuming that the membrane potential $V$ is given in units of $\mathrm{mV}$.

The steady-state inactivation function was defined directly as

$$
h_{\infty}=\frac{1}{1+\mathrm{e}^{(V+60) / 6.2}} .
$$

A fast potassium current, $I_{\mathrm{Kv}}$, was present in the soma $\left(\bar{g}_{\mathrm{Kv}}=95 \mathrm{pS} / \mu \mathrm{m}^{2}\right)$ and throughout the dendrites $\left(\bar{g}_{\mathrm{Kv}}=190 \mathrm{pS} / \mu \mathrm{m}^{2}\right)$,

$$
I_{\mathrm{Kv}}=\bar{g}_{\mathrm{Kv}} n\left(V-E_{\mathrm{K}}\right),
$$

with reversal potential $E_{\mathrm{K}}=-90 \mathrm{mV}$ and with activation gating variable $n$ 
characterized by

$$
\begin{aligned}
& \alpha_{\mathrm{n}}=-0.064 \frac{V-25}{\mathrm{e}^{-(V-25) / 9}-1} \\
& \beta_{\mathrm{n}}=0.0064 \frac{V-25}{\mathrm{e}^{(V-25) / 9}-1} .
\end{aligned}
$$

A high-voltage activated calcium current, $I_{\mathrm{Ca}}$, was distributed throughout the apical dendrites $\left(\bar{g}_{\mathrm{Ca}}=30 \mathrm{pS} / \mu \mathrm{m}^{2}\right)$ with an increased density $\left(\bar{g}_{\mathrm{Ca}}=35 \mathrm{pS} / \mu \mathrm{m}^{2}\right)$ for dendrites distal from the main apical dendrite's bifurcation,

$$
I_{\mathrm{Ca}}=\bar{g}_{\mathrm{Ca}} m^{2} h\left(V-E_{\mathrm{Ca}}\right),
$$

with reversal potential $E_{\mathrm{Ca}}=140 \mathrm{mV}$ and with activation gating variable $m$ and inactivation gating variable $h$ characterised by

$$
\begin{aligned}
\alpha_{\mathrm{m}} & =-0.177 \frac{V+27}{\mathrm{e}^{-(V+27) / 3.8}-1} \\
\beta_{\mathrm{m}} & =3.02 \mathrm{e}^{-(V+75) / 17} \\
\alpha_{\mathrm{h}} & =4.89 \cdot 10^{-4} \mathrm{e}^{-(V+13) / 50} \\
\beta_{\mathrm{h}} & =\frac{0.0071}{\mathrm{e}^{-(V+15) / 28}+1} .
\end{aligned}
$$

A calcium-dependent potassium current, $I_{\mathrm{KCa}}$, was similarly distributed throughout the apical dendrites $\left(\bar{g}_{\mathrm{KCa}}=30 \mathrm{pS} / \mu \mathrm{m}^{2}\right)$ with an increased density $\left(\bar{g}_{\mathrm{KCa}}=35 \mathrm{pS} / \mu \mathrm{m}^{2}\right)$ beyond the main bifurcation of the apical dendrite,

$$
I_{\mathrm{KCa}}=\bar{g}_{\mathrm{KCa}} n\left(V-E_{\mathrm{K}}\right)
$$

with activation gating variable $n$ characterised by

$$
\begin{aligned}
& \alpha_{\mathrm{n}}=0.032\left(\left[\mathrm{Ca}^{2+}\right]_{i}\right)^{6} \\
& \beta_{\mathrm{n}}=0.064
\end{aligned}
$$

with $\left[\mathrm{Ca}^{2+}\right]$ in $\mu \mathrm{M}$.

Internal calcium concentration in a shell below the membrane surface was computed 
using entry via $I_{\mathrm{Ca}}$ and removal by a first-order pump,

$$
\frac{\mathrm{d}\left[\mathrm{Ca}^{2+}\right]_{i}}{\mathrm{~d} t}=-\frac{10,000}{2 F d} I_{\mathrm{Ca}}+\frac{\left[\mathrm{Ca}^{2+}\right]_{\infty}-\left[\mathrm{Ca}^{2+}\right]_{i}}{\tau_{\mathrm{R}}}
$$

with Faraday constant $F$, depth of shell $d=0.1 \mu \mathrm{m}$ and with $\left[\mathrm{Ca}^{2+}\right]_{\infty}=0.1 \mu \mathrm{M}$, and $\tau_{\mathrm{R}}=80 \mathrm{~ms}$. To account for dendritic spines, the membrane capacitance and current densities were doubled throughout the dendrites. An axon was lacking in the cell reconstruction and was added as in Mainen and Sejnowski (1996).

Excitatory synaptic inputs were distributed over the membrane surface. Upon activation of a synapse, the conductance with a reversal potential of $0 \mathrm{mV}$ increased instantaneously and subsequently decayed exponentially with a time constant of $3 \mathrm{~ms}$. The $\mathrm{PP}_{\mathrm{CA} 1}$ provided 500 inputs that were distributed with uniform surface density throughout the distal apical tuft dendrites; the SC provided 2500 inputs, distributed uniformly over basal dendrites and proximal apical dendrites (Stuart et al., 2007).

All inputs were spatially tuned on a $2.5 \mathrm{~m}$ long linear track over which the simulated rat walked. The $\mathrm{PP}_{\mathrm{CA} 1}$ inputs showed periodic, grid field-like spatial tuning with periodicity ranging from 2 to 6 grid fields along the entire track with random phase: $G_{i}(x)=r \mathcal{H}\left(\cos \left(2 \pi k x+\xi_{i}\right)\right)$, where $\mathcal{H}$ is the Heaviside step function, $r$ is the mean firing rate within the grid field, $k$ is the spatial frequency, and $\xi_{i}$ is the random spatial phase offset for neuron $i$ (for $i=1, \ldots, 500$ ). The $2500 \mathrm{SC}$ inputs showed place field-like tuning, having single, $25 \mathrm{~cm}$ long place fields distributed uniformly random along the track. When the virtual rat was within the place or grid field of an $\mathrm{SC}$ or $\mathrm{PP}_{\mathrm{CA} 1}$ fiber, respectively, the input was activated as an independent Poisson process with a mean rate of $r=10$ spikes/s. Outside of the place/grid fields the fibers were quiescent. Simulations of the consolidation phase considered replay of the rat walking back and forth along the linear track, with running speeds increased, compared to realistic speeds, by a factor 20 $(5 \mathrm{~m} / \mathrm{s}$; Lee and Wilson (2002)). SC input activity to the CA1 cell was delayed by $5 \mathrm{~ms}$ with respect to the $\mathrm{PP}_{\mathrm{CA} 1}$ input (Yeckel and Berger, 1998), accounting for the extra processing stages involved for information reaching CA1 from the entorhinal cortex through DG and CA3, compared to the direct entorhinal $\mathrm{PP}_{\mathrm{CA} 1}$ input.

The $\mathrm{PP}_{\mathrm{CA} 1}$ and/or SC inputs showed additive STDP, operating in the same manner as defined in the Methods for Fig. 1. (Eq. (44). Post-synaptic spikes were defined as 
local voltage crossings of a threshold at $-30 \mathrm{mV}$. The maximum synaptic weight for the $\mathrm{SC}$ inputs was $400 \mathrm{pS}$ and $140 \mathrm{pS}$ for the PP $\mathrm{CA} 1_{\text {inputs. }}$

The reference tuning curve shown in Fig. $2 \mathrm{~F}$ ( $\mathrm{PP}_{\mathrm{CA} 1}$ inputs theory) was computed by adding up all grid field tuning functions that had an active field in the SC-encoded spatial position (i.e., halfway along the linear track).

Simulations were carried out with a fixed time step of $25 \mu$ s using the NEURON simulation software (Hines and Carnevale, 1997).

\section{Consolidation of place-object associations in multiple hippocampal stages}

The Results related to Fig. 3 show the acquisition and consolidation of place-object associations in a hippocampal network model. Every day a virtual animal learns the position of one of many possible objects in a circular open field environment. The simulations show that during a subsequent sleep phase, replay of the hippocampal activity that is associated with runs through this environment allows for the consolidation of the place-object association. We call the imprinting of a new memory and the subsequent memory consolidation phase a consolidation cycle. In the simulations, a place-object association learned at time $t=0$ is tracked for $N_{\text {cycle }}$ consolidation cycles, i.e., nights after memory acquisition. Between consolidation cycles, the memory in the system is assessed as described below.

\section{Model architecture}

The model consists of four neuronal layers: entorhinal cortex (EC), dentate gyrus/CA3 (DG-CA3; note that the dentate gyrus is not explicitly included as a separate area), CA1, and the subiculum (SUB). Each layer consists of a population of place-coding cells and a population of object-coding cells. The connectivity is depicted in Fig. 3A: EC projects to DG-CA3, which connects to CA1 (through the SC pathway), which in turn

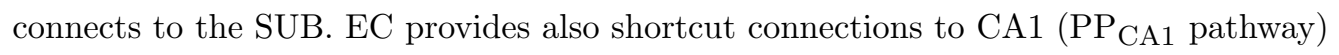
and the SUB (PPSUB pathway).

The $\mathrm{SC}, \mathrm{PP}_{\mathrm{CA} 1}$, and $\mathrm{PP}_{\mathrm{SUB}}$ pathways consist of four different connection types among populations of neurons that represent either place or object: (i) from object 
(populations) to object (populations), (ii) from place to place, (iii) from object to place, and (iv) from place to object. For simplicity, the pathway from CA1 to the SUB consists only of place-to-place and object-to-object connections, because we never store object-place or place-object associations in this pathway. The pathway from EC to DG-CA3 was not explicitly modelled. Instead, we assumed that the same location (of the virtual animal) is represented in both areas, but with a grid cell code and a place cell code, respectively. We assumed that all connections have the same transmission delay, which is equal to one time step $D=\Delta T=5 \mathrm{~ms}$ in the simulation (see Table 1 for parameter values). In practise, this meant that the activities in the SC pathway and the connection from CA1 to the SUB each had a transmission delay $D$ relative to the activities in the connections from EC to CA1 and from EC to SUB.

Activities of neurons in each layer were described as firing rates and were determined by a linear model,

$$
\begin{aligned}
& \mathbf{y}_{\mathrm{CA} 1}(t)=\mathbf{W}_{\mathrm{PP}-\mathrm{CA} 1}^{\top}(t) \mathbf{x}_{\mathrm{EC}}(t)+\mathbf{V}_{\mathrm{SC}}^{\top} \mathbf{x}_{\mathrm{CA} 3}(t-D), \\
& \mathbf{y}_{\mathrm{SUB}}(t)=\mathbf{W}_{\mathrm{PP}-\mathrm{SUB}}^{\top}(t) \mathbf{x}_{\mathrm{EC}}(t)+\mathbf{V}_{\mathrm{CA} 1-\mathrm{SUB}}^{\top} \mathbf{y}_{\mathrm{CA} 1}(t-D),
\end{aligned}
$$

where $\mathbf{x}_{\mathrm{EC}}(t)$ and $\mathbf{x}_{\mathrm{CA} 3}(t)$ are the activities in the input layers EC and DG-CA3, respectively, and $\mathbf{y}_{\mathrm{CA} 1}(t)$ and $\mathbf{y}_{\mathrm{SUB}}(t)$ represent the activities in the output layers CA1 and SUB, respectively. Time is denoted by $t$. The symbols $\mathbf{W}_{\text {PP-CA1 }}$ and $\mathbf{W}_{\text {PP-SUB }}$ denote the weight matrices of the pathways from EC to CA1 and from EC to SUB, respectively. The matrices $\mathbf{V}_{\mathrm{SC}}$ and $\mathbf{V}_{\mathrm{CA1} \text {-SUB }}$ summarise the weights from DG-CA3 to CA1 and from CA1 to SUB, respectively, which mediate the transmission delay $D$.

As already mentioned above, each neuron in a layer is assumed to primarily encode either place or object information (see Fig. 3AA). To simplify the mathematical analysis, we turn to a notation where we write a layer's activity vector $\mathbf{z}$ (where $\mathbf{z}=\mathbf{x}_{\mathrm{EC}}, \mathbf{x}_{\mathrm{CA} 3}, \mathbf{y}_{\mathrm{CA} 1}$, or $\left.\mathbf{y}_{\mathrm{SUB}}\right)$ as a concatenation of place and object vectors:

$$
\mathbf{z}=\left[\begin{array}{c}
\mathbf{z}^{\text {place }} \\
\mathbf{z}^{\text {object }}
\end{array}\right]
$$

where the number of place- and object-coding cells is identical, 
$\operatorname{dim}\left(\mathbf{z}^{\text {place }}\right)=\operatorname{dim}\left(\mathbf{z}^{\text {object }}\right)=N$, hence $\operatorname{dim}(\mathbf{z})=2 N$. Correspondingly, the weight matrices $\mathbf{M}$ (where $\mathbf{M}=\mathbf{W}_{\mathrm{PP}-\mathrm{CA} 1}, \mathbf{W}_{\mathrm{PP}-\mathrm{SUB}}, \mathbf{V}_{\mathrm{SC}}$, or $\mathbf{V}_{\mathrm{CA} 1-\mathrm{SUB}}$ ) are composed of four submatrices, connecting the corresponding feature encoding sub-vectors (place-place, place-object, object-place, and object-object):

$$
\mathbf{M}=\left[\begin{array}{cc}
\mathbf{M}^{\text {place,place }} & \mathbf{M}^{\text {object,place }} \\
\mathbf{M}^{\text {place,object }} & \mathbf{M}^{\text {object,object }}
\end{array}\right]
$$

Associations between objects and places were initially stored in $\mathbf{V}_{\mathrm{SC}}$ as described below. To achieve a consistency in the code for places and objects, the weights in $\mathbf{V}_{\mathrm{SC}}$ and $\mathbf{V}_{\mathrm{CA1} \text {-SUB }}$ that connect neurons coding for the same feature (i.e., place-place or object-object) were set proportional to identity matrices $\mathbf{I}$,

$$
\begin{aligned}
& \mathbf{V}_{\mathrm{SC}}^{\text {place,place }}=\mathbf{V}_{\mathrm{SC}}^{\text {object,object }}=w_{\mathrm{SC}}^{\mathrm{id}} \mathbf{I} \\
& \mathbf{V}_{\mathrm{SC}}^{\text {place,place }}=\mathbf{V}_{\mathrm{CA1-SUB}}^{\text {object,object }}=w_{\mathrm{CA} 1-\mathrm{SUB}}^{\mathrm{id}} \mathbf{I} .
\end{aligned}
$$

The scaling factors $w_{\mathrm{SC}}^{\mathrm{id}}=\frac{1}{4}$ and $w_{\mathrm{CA} 1-\mathrm{SUB}}^{\mathrm{id}}=\frac{1}{2}$ ensure that these pathways had similar impact as the other pathways projecting to CA1 cells and SUB cells, respectively, and $w_{\mathrm{CA} 1-\mathrm{SUB}}^{\mathrm{id}}$ is twice as large as $w_{\mathrm{SC}}^{\mathrm{id}}$ to account for the fact that only in the CA1-SUB pathway the object-place and place-object connections were set to zero. The matrices $\mathbf{W}_{\text {PP-CA1 }}$ and $\mathbf{W}_{\text {PP-SUB }}$, which represent shortcuts, were plastic during a consolidation cycle and evolved according to the learning rule described below. Their initial values were chosen as a random permutation of an equilibrium state, taken from a long running previous simulation.

\section{Place- and object-coding cells}

Place-coding cells in EC and DG-CA3 were assumed to respond deterministically, given a two-dimensional position variable $\mathbf{p}(t) \in[0,1]^{2}$, which evolves in time.

Place-coding cells in entorhinal cortex show grid field spatial tuning (Moser et al. 2008 ), which we modelled as a superposition of 3 plane waves with relative angles of $\frac{\pi}{3}$ :

$$
\mathbf{x}_{\mathrm{EC}, i}^{\text {place }}(t)=r_{\max } \frac{2}{9} \sum_{l=1}^{3}\left[\frac{1}{2}+\cos \left(m_{i} \mathbf{k}_{i}^{l}\left(\mathbf{p}(t)-\mathbf{p}_{i}\right)\right)\right]
$$


where the spacing $m_{i}=2 \pi\left(2+\frac{4 i}{N}\right), \forall i \in[1, N]$, is chosen so that a total range of 2 to 6 periods fit into the circular environment. The orientation of the plane waves is determined by the vector $\mathbf{k}_{i}^{l}=\left[\cos \left(l \frac{\pi}{3}+\theta_{i}\right), \sin \left(l \frac{\pi}{3}+\theta_{i}\right)\right]$ where $\theta_{i}$ are uniformly chosen random angles, and $\mathbf{p}_{i} \in[0,1]^{2}$ are uniformly sampled random phases of the grid field Solstad et al. 2006). Each cell's output rate varies between 0 to $r_{\max }$ spikes per second.

Place-coding cells in DG-CA3 show place-field tuning and were assumed to have a

2D Gaussian activity profile

$$
\mathbf{x}_{\mathrm{CA} 3, i}^{\text {place }}(t)=r_{\max } \exp \left(-\frac{\left(\mathbf{p}(t)-\mathbf{c}_{i}\right)^{2}}{2 \sigma^{2}}\right)
$$

where $r_{\max }$ is the maximum rate, $\sigma$ the field size, and $\mathbf{c}_{i}$ the centre of field $i$. The centres $\mathbf{c}_{i}$ were chosen to lie on a regular grid.

The object-coding cells in EC and DG-CA3 respond with fixed deterministic responses $\mathbf{x}_{\mathrm{EC}}^{\text {object }}$ and $\mathbf{x}_{\mathrm{CA} 3}^{\text {object }}$ to each of $N_{\text {object }}$ objects. Given that they are located in the same brain region, we assumed that the firing-rate statistics of the object-coding cells and the place-coding cells were similar, both in EC and CA1. This was ensured by calculating the rates of the object-coding cells in two steps. First, we used the same equations as for the place-coding cells (i.e., Eq. 21) for EC cells and Eq. 222) for DG-CA3 cells) with a randomly selected "object position" $\mathbf{o}_{i}, i \in\left\{1, . ., N_{\text {object }}\right\}$ for each of the $N_{\text {object }}$ objects. Subsequently the rates of the neurons within the population were randomly permuted for each object, to avoid an artificial constraint of the population activity onto a 2-dimensional manifold.

\section{Imprinting of place-object associations in the SC pathway}

The virtual animal learned a single new object-to-place association each day. Storing more memories per day would not qualitatively change the results, but would merely alter the time scale at which a given memory is overwritten in the SC pathway.

Memories were imprinted in $\mathbf{V}_{\mathrm{SC}}$ by first determining the activities of the object-coding DG-CA3 cells and place-coding CA1 cells given a random object and a random position where the object was encountered (see previous section). The weights in $\mathbf{V}_{\mathrm{SC}}$ that 
connect object cells to place cells were then updated according to

$$
\mathbf{V}_{\mathrm{SC}} \leftarrow\left[\mathbf{V}_{\mathrm{SC}}+\frac{\lambda_{\mathrm{SC}}\left[\mathbf{x}_{\mathrm{CA} 3} \mathbf{y}_{\mathrm{CA} 1}^{\mathrm{T}}\right]^{\text {norm }}}{1-\lambda_{\mathrm{SC}}}\right]^{\text {norm }}
$$

where $0<\lambda_{\mathrm{SC}}<1$ (numerical values of parameters are summarised in Table 1 ) denotes the strength of the new memory and controls the rate of forgetting. The symbol $[\mathbf{M}]^{\text {norm }}$ denotes the normalised version of the matrix $\mathbf{M}$; the normalisation ensures that the biggest sum along the columns of $[\mathbf{M}]^{\text {norm }}$ was 1 by rescaling all entries of $\mathbf{M}$ with the same factor. The specific choice of the normalisation does not alter the results. The inner norm in Eq. 23 ensures the same relative influence of different memories, irrespective of the associated activity levels. This ensures an approximately constant rate of overwriting/forgetting. The outer norm guarantees that the weights $\mathbf{V}_{\mathrm{SC}}$ stay bounded and hence induces forgetting. As a consequence of this updating scheme, the memories are lost over time. Note that before we imprint a new memory to $\mathbf{V}_{\mathrm{SC}}$ (other than on day 0 on which the place-object association is learned that is tracked during the simulation), the place-coding cells in DG-CA3 are remapped, i.e., they are assigned to new random positions. This corresponds to learning the new object in a new environment/room, and effectively reduces the amount in interference between memories. Before starting a simulation, we imprinted $N_{\text {mem }}$ place-object associations to $\mathbf{V}_{\mathrm{SC}}$ to ensure an equilibrium state.

The weights from place-to-object coding cells could be updated analogously. This would allow to decode the identity of a stored object given a location. We did not test this direction of the object-place association, because this is not relevant for the water maze task.

\section{Learning rule operating on $\mathrm{PP}_{\mathrm{CA} 1}$ and $\mathrm{PP}_{\mathrm{SUB}}$ pathways}

The plastic weight matrices $\mathbf{W}_{\text {PP-CA1 }}$ and $\mathbf{W}_{\text {PP-SUB }}$ changed according to a timing-based learning rule (Dayan and Abbott, 2001):

$$
\frac{\mathrm{d} \mathbf{W}}{\mathrm{d} t}=\int_{0}^{\infty} \mathrm{d} \tau\left[L(\tau) \mathbf{x}_{\mathrm{EC}}(t-\tau) \mathbf{y}^{\top}(t-D)+L(-\tau) \mathbf{x}_{\mathrm{EC}}(t) \mathbf{y}^{\top}(t-D-\tau)\right]
$$


where $\mathbf{W}$ is either $\mathbf{W}_{\mathrm{PP}-\mathrm{CA} 1}$ or $\mathbf{W}_{\mathrm{PP}-\mathrm{SUB}}$, and $\mathbf{y}$ correspondingly $\mathbf{y}_{\mathrm{CA} 1}$ or $\mathbf{y}_{\mathrm{SUB}}$. The learning window $L(\tau)$ (see Eq. (4) ) determines the learning dynamics.

We integrated the learning dynamics using the Euler method, with time steps $\Delta T$ equal to the inverse pattern presentation rate. In practise, we used the standard method of calculating pre- and postsynaptic traces $\hat{\mathbf{x}}$ and $\hat{\mathbf{y}}$ to integrate the equation

$$
\frac{\mathrm{d} \mathbf{W}}{\mathrm{d} t}=A^{+} \hat{\mathbf{x}}_{\mathrm{EC}}(t) \mathbf{y}^{\top}(t-D)+A^{-} \mathbf{x}_{\mathrm{EC}}(t) \hat{\mathbf{y}}^{\top}(t-D)
$$

where $A^{+}$and $A^{-}$again determine the maximum amount of potentiation and depression of the synaptic weights, respectively. Note that these parameters effectively control the learning rate and are chosen twice as large in the $\mathrm{PP}_{\mathrm{CA} 1}$ than in the $\mathrm{PP}_{\mathrm{SUB}}$ (Table 1), to increase memory lifetime in the latter shortcut. Again, we used an exponential window function $L(\tau)$, so that exponentially filtered activities $\hat{\mathbf{x}}$ and $\hat{\mathbf{y}}$ can be calculated as in Song et al. (2000):

$$
\tau_{\mathrm{STDP}} \frac{\mathrm{d} \hat{\mathbf{x}}_{\mathrm{EC}}(t)}{\mathrm{d} t}=\mathbf{x}_{\mathrm{EC}}(t)-\hat{\mathbf{x}}_{\mathrm{EC}}(t) \quad \text { and } \quad \tau_{\mathrm{STDP}} \frac{\mathrm{d} \hat{\mathbf{y}}(t)}{\mathrm{d} t}=\mathbf{y}(t)-\hat{\mathbf{y}}(t)
$$

where $\tau_{\mathrm{STDP}}$ determines the width of the learning window.

Weight values are constrained to the interval $\left[0, w_{\max }\right]$. The weights of $\mathbf{W}_{\mathrm{PP}-\mathrm{CA} 1}$ and $\mathbf{W}_{\text {PP-SUB }}$ were initialised to small random values from a uniform distribution in $\left[0, w_{\text {init }}^{\max }\right]$.

For each iteration in a consolidation cycle of duration $T$, i.e., every $\Delta t=5 \mathrm{~ms}$, we chose a random input position and a random object to calculate the activities in all layers. These activities were then used to update the weights as given in Eq. 25.

\section{Assessing the strength of memories in $\mathrm{SC}, \mathrm{PP}_{\mathrm{CA} 1}$, and $\mathrm{PP}_{\mathrm{SUB}}$}

To assess the memory strength encoded in a pathway, we determine the activity $\mathbf{y}^{\text {place }}$ of place-coding cells (in either CA1 or SUB) in response to an object $o \in\left\{1, \ldots, N_{\text {objects }}\right\}$ along the object-to-place pathway under consideration (e.g., for $\mathrm{PP}_{\mathrm{CA} 1}$ it would be from object-coding cells in EC to place-coding cells in CA1). From this response we decode the memorised place of the object using Bayesian inference. However, the response is usually corrupted due to various factors such as imperfect imprinting, consolidation, or 
interference with other memories. Assuming that these imperfections result from a superposition of many statistically independent factors, we use a Gaussian likelihood:

$$
p\left(\mathbf{y}^{\text {place }} \mid \mathbf{p}\right)=\mathcal{N}\left(\mu(\mathbf{p}), \sigma_{\text {noise }} \mathbf{I}\right),
$$

where $\mathcal{N}$ is the multivariate Gaussian probability density function, $\sigma_{\text {noise }}$ is the standard deviation of the noise, i.e., the imperfections. I is the identity matrix, i.e., we assumed uncorrelated noise in the responses.

The expected activity $\mu(\mathbf{p})$ depends on the location $\mathbf{p}$ and is given by the activity that would result from the activation of place-coding cells in EC or DG-CA3, i.e., by Eqs. 22, 15, 16. Because the connections between place-coding cells in DG-CA3, CA1, and SUB are scaled identity matrices, the expected activity $\mu(\mathbf{p})$ is essentially a place-cell code:

$$
\mu(\mathbf{p}) \propto \mathbf{x}_{\mathrm{CA} 3}^{\text {place }}(\mathbf{p})
$$

To avoid a dependence on overall activity levels, $\mu(\mathbf{p})$ and $\mathbf{y}^{\text {place }}$ are normalised to zero mean and unit variance.

Using Bayes' theorem we can now calculate the posterior probabilities of the places that coded for the given response $\mathbf{y}^{\text {place }}$ :

$$
\begin{aligned}
p\left(\mathbf{p} \mid \mathbf{y}^{\text {place }}\right) & =\frac{p\left(\mathbf{y}^{\text {place }} \mid \mathbf{p}\right) p(\mathbf{p})}{\sum_{\mathbf{p}} p\left(\mathbf{y}^{\text {place }} \mid \mathbf{p}\right) p(\mathbf{p})} \\
& =\frac{p\left(\mathbf{y}^{\text {place }} \mid \mathbf{p}\right)}{\sum_{\mathbf{p}} p\left(\mathbf{y}^{\text {place }} \mid \mathbf{p}\right)} \\
& \propto \exp \left(\frac{-\left(\mathbf{y}^{\text {place }}-\mu(\mathbf{p})\right)^{2}}{2 \sigma_{\text {noise }}^{2}}\right),
\end{aligned}
$$

where for Eq. (30) we used a flat prior, because the environment was uniformly sampled in the simulations. To avoid the explicit evaluation of the sum in the denominator, we normalise the evaluated place probabilities to sum to one. We make use of the linear relationship of the place response given an object (see Eqs. 15 to 18):

$$
\mathbf{y}^{\text {place }}(o)=\left(\mathbf{M}^{\text {object, place }}\right)^{\top} \mathbf{x}^{\text {object }}(o)
$$


where the matrix $\mathbf{M}^{\text {object,place }}$ is either $\mathbf{V}_{\mathrm{SC}}^{\text {object,place }}, \mathbf{W}_{\mathrm{PP}-\mathrm{CA} 1}^{\text {object,place }}$, or $\mathbf{W}_{\mathrm{PP}-\mathrm{SUB}}^{\text {object,place }}$, depending on the pathway for which the strength of the memory is assessed. This allows to compute the posterior probability of the place given an object (Fig. 3 B, C):

$$
p(\mathbf{p} \mid o) \propto \exp \left(\frac{-\left(\mathbf{y}^{\text {place }}(o)-\mu(\mathbf{p})\right)^{2}}{2 N \sigma_{\text {noise }}^{2}}\right)
$$

\section{Memory consolidation over many days}

To simulate a single consolidation cycle (i.e., a storage of a new memory followed by a single consolidation phase), we alternated the imprinting of a new place-object association (Eq. 23) ) with a consolidation phase of length T. Before starting the experiments, we equilibrated the weights $\mathbf{W}_{\text {PP-CA1 }}$ and $\mathbf{W}_{\text {PP-SUB }}$ by simulating $N_{\text {equi }}$ consolidation cycles. At day 0 we imprinted the object $\hat{o}$ the memory of which was tracked. After each following consolidation phase the place probabilities along the different pathways were calculated for object $\hat{o}$ according to Eq. (33) (see Fig. $3 \mathrm{C}$ of main text).

\section{Lesion experiments}

Remondes and Schuman (2004) lesioned the perforant path (temporoammonic pathway) during a Morris water maze consolidation experiment. Their finding evidenced a role of the perforant path in memory consolidation by showing that the precise time-point of the lesion after memory acquisition determined whether the memory persisted (see Fig. 3D of main text).

In our simulations we implemented a lesion by setting all PP $\mathrm{CA}_{1}$ weights to 0 $\left(\mathbf{W}_{\mathrm{PP}-\mathrm{CA} 1}=0\right)$ and by disabling their plasticity. Like in the experimental setup of Remondes and Schuman (2004), we lesioned either right before or 21 days after presentation of object $\hat{o}$. For each day and lesioning protocol, the place probabilities, Eq. (33), along the pathways can then be calculated. The pathway with the highest inferred object position probability was then selected, and the summed probabilities per quadrant were calculated for this pathway. To account for exploration versus exploitation (see, e.g., Sutton and Barto (1998)) of the rats, the inferred probabilities were linearly mixed with a uniform distribution over the quadrants. We used $70 \%$ explore versus $30 \%$ exploit for the plots in Fig. 3D. Note that we assumed that the 


\begin{tabular}{|c|c|c|}
\hline$N_{\text {cycle }}$ & 31 & number of consolidation cycles \\
\hline$T$ & $150 \mathrm{~s}$ & consolidation time per sleep cycle \\
\hline$\Delta T$ & $5 \mathrm{~ms}$ & integration time step \\
\hline$N$ & 256 & neurons per place- or object-coding population \\
\hline$N_{\text {object }}$ & 128 & number of different objects \\
\hline$r_{\max }$ & 10 spikes/s & maximum output firing rate \\
\hline$\sigma$ & 0.1 & size of place field standard deviation \\
\hline$D$ & $5 \mathrm{~ms}$ & transmission delay \\
\hline$w_{\mathrm{SC}}^{\mathrm{id}}$ & $\frac{1}{4}$ & $\begin{array}{l}\text { weight between object-object and place-place coding } \\
\text { cells in DG-CA3 and CA1 }\end{array}$ \\
\hline$w_{\mathrm{CA} 1-\mathrm{SUB}}^{\mathrm{id}}$ & $\frac{1}{2}$ & $\begin{array}{l}\text { weight between object-object and place-place coding } \\
\text { cells in CA1 and SUB }\end{array}$ \\
\hline$\lambda_{\mathrm{SC}}$ & 0.6 & $\begin{array}{l}\text { relative strength of new place-object association in } \\
\mathbf{V}_{\mathrm{SC}}\end{array}$ \\
\hline$N_{\text {mem }}$ & 125 & number of associations stored to initialize $\mathbf{V}_{\mathrm{SC}}$ \\
\hline$w_{\max }$ & $\frac{1}{N}$ & maximum weight values for $\mathbf{W}_{\mathrm{PP}-\mathrm{CA} 1}$ and $\mathbf{W}_{\mathrm{PP} \text {-SUB }}$ \\
\hline$w_{\text {init }}^{\max }$ & $\frac{1}{10} \cdot w_{\max }$ & $\begin{array}{l}\text { maximum initial weight values for } \mathbf{W}_{\text {PP-CA1 }} \text { and } \\
\mathbf{W}_{\text {PP-SUB }}\end{array}$ \\
\hline$A_{\mathrm{PP}-\mathrm{CA} 1}^{+}$ & $0.05 \cdot w_{\max }$ & height of potentiating learning window for $\mathbf{W}_{\text {PP-CA1 }}$ \\
\hline$A_{\mathrm{PP}-\mathrm{CA} 1}^{-}$ & $-1.00025 \cdot A_{\mathrm{PP}-\mathrm{CA} 1}^{+}$ & height of depressing learning window for $\mathbf{W}_{\text {PP-CA1 }}$ \\
\hline$A_{\mathrm{PP}-\mathrm{SUB}}^{+}$ & $0.5 \cdot A_{\mathrm{PP}-\mathrm{CA} 1}^{+}$ & height of potentiating learning window for $\mathbf{W}_{\text {PP-SUB }}$ \\
\hline$A_{\mathrm{PP}-\mathrm{SUB}}^{-}$ & $0.5 \cdot A_{\mathrm{PP}-\mathrm{CA} 1}^{-}$ & height of depressing learning window for $\mathbf{W}_{\text {PP-SUB }}$ \\
\hline$\tau_{\mathrm{STDP}}$ & $20 \mathrm{~ms}$ & time constants of learning window \\
\hline$N_{\text {equi }}$ & 10 & $\begin{array}{l}\text { equilibration sleep phases run before the simulation } \\
\text { starts }\end{array}$ \\
\hline$\sigma_{\text {noise }}$ & 4.8 & noise level assumed for place inference \\
\hline
\end{tabular}

Table 1. Parameters for simulations shown in Fig, 3

probabilities per quadrant correspond to the time spent in each quadrant. 


\section{Consolidation in a hierarchical rate-based network}

The model used in Fig. 4 of the main text demonstrates the consolidation of memories in a hierarchy of connected neural populations (see Fig. 44A). In the model, signals flow along distinct neocortical neural populations to the hippocampal formation (HPC) and back into neocortex (black arrows in Fig. $4 \mathrm{~A}$ ). Shortcut connections exist between the neocortical populations (colored arrows in Fig. 44). All connections carry the same transmission delay $D$.

Every day new memories are imprinted into the weight matrix connecting the neocortex to the HPC. The model describes the transfer of the memories into neocortex during $N_{\text {cycle }}$ consolidation phases, of which there is one per night. In contrast to the model for Fig. 3, we do not consider object-place associations, but directly analyse correlations between a stored memory weight matrix and the weight matrices that describe the neocortical shortcut connections.

\section{Model details}

We consider a hierarchy of $2 L$ neocortical populations with $L$ shortcut connections. Activities of the populations that project towards the HPC are given by $\mathbf{x}_{i}(t)$ and the activities of the populations leading away from the HPC by $\mathbf{y}_{i}(t)(i \in\{1, \ldots, L\})$. At each iteration, the activities $\mathbf{x}_{L}(t)$ (i.e., the neocortical population most distal from the HPC) are sampled from a Gaussian distribution with a mean input rate $r$ (see Table 2 ) and a standard deviation $r / 2$. The sampled activities are rectified to be non-negative $(r \rightarrow \max (r, 0))$, hence yielding a rectified Gaussian distribution. The activities on all other layers are then determined by their respective connections. For simplicity, we assume that weight matrices connecting subsequent populations in the hierarchy (black arrows in Fig. 4A) are identity matrices that are scaled such that activity levels remain comparable along the hierarchy (see below). The results do not depend on this simplifying assumption. The population activities along the HPC directed path are then given as

$$
\mathbf{x}_{i}=\mathbf{x}_{i+1}(t-D), \quad \forall i \in\{1, . ., L-1\}
$$


In Fig. 4, we modelled the HPC as a single neural population, with activities given by

$$
\mathbf{y}_{\mathrm{HPC}}(t)=\mathbf{W}_{\mathrm{HPC}}^{T} \mathbf{x}_{1}(t-D)
$$

Here, $\mathbf{W}_{\mathrm{HPC}}$ is the hippocampal-formation weight matrix into which new memories are imprinted (see below).

The first outward-directed neocortical population receives input from the HPC and through a shortcut connection from the activities $\mathbf{x}_{1}$,

$$
\mathbf{y}_{1}(t)=\frac{1}{2} \mathbf{W}_{1}^{T} \mathbf{x}_{1}(t-D)+\frac{1}{2} \mathbf{y}_{\mathrm{HPC}}(t-D)
$$

and subsequent activities $\mathbf{y}_{i}$ of populations projecting away from HPC are calculated in analogy to the previous section as

$$
\mathbf{y}_{i}(t)=\frac{1}{2} \mathbf{W}_{i}^{T} \mathbf{x}_{i}(t-D)+\frac{1}{2} \mathbf{y}_{i-1}(t-D), \quad \forall i \in\{2, . ., L\}
$$

where $\mathbf{W}_{i}$ are the direct shortcut connections from the populations $\mathbf{x}_{i}$ to the populations $\mathbf{y}_{i}$. Activities were updated in the order given by the black arrows in Fig. 44 4 , i.e., starting with $\mathbf{x}_{L}$ to $\mathbf{x}_{1}$, then $\mathbf{y}_{\mathrm{HPC}}$, and afterwards $\mathbf{y}_{1}$ to $\mathbf{y}_{L}$.

Memory imprinting to the HPC weight matrix $\mathbf{W}_{\mathrm{HPC}}$ is analogous to the section for Fig. 3 (compare Eq. 23p). Before each consolidation phase, new memories were sampled from a binomial distribution $\mathbf{B}(1,0.5)$. The HPC weights were then updated as

$$
\mathbf{W}_{\mathrm{HPC}} \leftarrow\left[\mathbf{W}_{\mathrm{HPC}}+\frac{\lambda[\mathbf{B}(1,0.5)]_{1}^{\text {norm }}}{1-\lambda}\right]_{1}^{\text {norm }}
$$

where $[\mathbf{M}]_{1}^{\text {norm }}$ now denotes the L1 normalization of the matrix $\mathbf{M}$.

All shortcut connections $\mathbf{W}_{i}$ showed plasticity according to Eqs. 25) and 26] with parameters $A_{i}^{+}$and $A_{i}^{-}$specified in Table 2 Weights were constrained to the interval $\left[0, w_{\max }\right]$ with $w_{\max }=\frac{2}{N}$ and $N$ being the number of neurons per layer. Initial weights were drawn from a uniform distribution in this interval. To increase memory lifetime in the system, learning rates were decreased along the hierarchy, such that the learning rate in layer $i$ is smaller than that in layer 1 by a factor $q^{i-1}$. Hence, layers closer to the HPC are more plastic than more remote layers. 


\begin{tabular}{|c|c|c|}
\hline$N_{\text {cycle }}$ & 1000 & number of consolidation cycles \\
\hline$T$ & $150 \mathrm{~s}$ & consolidation time per sleep cycle \\
\hline$\Delta T$ & $5 \mathrm{~ms}$ & integration time step \\
\hline$N$ & 256 & neurons per neuron population \\
\hline$L$ & 8 & number of neocortical populations \\
\hline$r$ & 10 spikes/s & mean firing rate \\
\hline$D$ & $5 \mathrm{~ms}$ & transmission delay \\
\hline$\lambda$ & 0.5 & $\begin{array}{l}\text { relative strength of new memory to HPC weights (see } \\
\text { eq } 38\end{array}$ \\
\hline$w_{\max }$ & $2 / N$ & maximum weight \\
\hline$\overline{A_{i}^{+}}$ & $0.4 \cdot w_{\max } \cdot q^{i-1}$ & $\begin{array}{l}\text { height of potentiating learning window for connec- } \\
\text { tions between populations at level } i\end{array}$ \\
\hline$A_{i}^{-}$ & $-1.00008 \cdot A_{i}^{+}$ & $\begin{array}{l}\text { height of depressing learning window for connections } \\
\text { between populations at level } i\end{array}$ \\
\hline$q$ & 0.5 & learning rate decrease factor \\
\hline$\tau_{\mathrm{STDP}}$ & $20 \mathrm{~ms}$ & time constants of learning window (see eq 26 ) \\
\hline$N_{\text {equi }}$ & 1000 & $\begin{array}{l}\text { equilibration consolidation cycles run before the sim- } \\
\text { ulation starts }\end{array}$ \\
\hline
\end{tabular}

Table 2. Parameters for simulations in Fig. 4

Before starting the main simulation of $N_{\text {cycle }}$ consolidation cycles, we equilibrated the weight matrices by simulating $N_{\text {equi }}$ consolidation cycles.

\section{Assessing the strength of memories in neocortical weight matrices}

To assess the decay of memory in the system, a reference memory $\mathbf{W}_{\text {ref }}$ was imprinted according to Eq. (38) to $\mathbf{W}_{\text {HPC }}$ at time $t=0$. The memory pathway correlation, i.e., the Pearson correlation of this reference memory with all shortcut weight matrices $\mathbf{W}_{i}$ was then calculated.

In analogy to the section for Fig. 3. the maximum correlation (across layers) was taken as the overall memory signal of the system. This yields the power law in Fig. $4 \mathrm{~B}$. The noise level indicated in Fig. $4 \mathrm{~B}$ is the standard deviation of the correlation between the two random matrices $\mathbf{M}$ and $\mathbf{W}_{\text {ref }}$, both having sample size $N^{2}$. Considering the central limit theorem, the noise level will be approximately $1 / N$. 


\section{Code availability}

The code will be made available upon publication.

\section{References}

Cannon, R. C., D. A. Turner, G. K. Pyapali, and H. V. Wheal, An on-line archive of reconstructed hippocampal neurons, Journal of Neuroscience Methods, 84(1-2), $49-54,1998$.

Dayan, P., and L. F. Abbott, Theoretical Neuroscience, MIT Press, Cambridge, 2001.

Hines, M. L., and N. T. Carnevale, The NEURON simulation environment, Neural Computation, 9(6), 1179-1209, 1997.

Lee, A., and M. Wilson, Memory of sequential experience in the hippocampus during slow wave sleep, Neuron, 36(6), 1183-1194, 2002.

Mainen, Z. F., and T. J. Sejnowski, Influence of dendritic structure on firing pattern in model neocortical neurons, Nature, 382(6589), 363-366, 1996.

Moser, E. I., E. Kropff, and M.-B. Moser, Place cells, grid cells, and the brain's spatial representation system, Annual Review of Neuroscience, 31, 69-89, 2008.

Remondes, M., and E. Schuman, Role for a cortical input to hippocampal area CA1 in the consolidation of a long-term memory, Nature, 431(7009), 699-703, 2004.

Solstad, T., E. I. Moser, and G. T. Einevoll, From grid cells to place cells: a mathematical model, Hippocampus, 16 (12), 1026-1031, 2006.

Song, S., K. D. Miller, and L. F. Abbott, Competitive hebbian learning through spike-timing-dependent synaptic plasticity, Nature Neuroscience, 3(9), 919-926, 2000.

Stuart, G., N. Spruston, and M. Häusser, Dendrites, Oxford University Press, Oxford, 2007.

Sutton, R. S., and A. G. Barto, Introduction to Reinforcement Learning, MIT Press, Cambridge, 1998. 

available under aCC-BY 4.0 International license.

Troyer, T. W., and K. D. Miller, Physiological gain leads to high isi variability in a simple model of a cortical regular spiking cell, Neural Computation, 9(5), 971-983, 1997.

Yeckel, M. F., and T. W. Berger, Spatial distribution of potentiated synapses in hippocampus: dependence on cellular mechanisms and network properties, Journal of Neuroscience, 18(1), 438-450, 1998. 


\title{
SUPPLEMENTARY MATERIAL
}

\section{Hebbian plasticity in parallel synaptic pathways: A circuit mechanism for systems memory consolidation}

\author{
Michiel Remme ${ }^{1 \dagger}$, Urs Bergmann ${ }^{1 \dagger}$, Denis Alevi ${ }^{2,3}$, Susanne Schreiber ${ }^{1,3,4}$, Henning \\ Sprekeler ${ }^{2,3,4,5+}$, Richard Kempter ${ }^{1,3,4,+, *}$ \\ ${ }^{1}$ Department of Biology, Institute for Theoretical Biology, Humboldt-Universität zu \\ Berlin, Phillipstr. 13, 10115 Berlin, Germany \\ ${ }^{2}$ Department for Electrical Engineering and Computer Science, Technische Universität \\ Berlin, Marchstr. 23, 10587 Berlin, Germany \\ ${ }^{3}$ Bernstein Center for Computational Neuroscience Berlin, Philippstr. 13, 10115 Berlin, \\ Germany \\ ${ }^{4}$ Einstein Center for Neurosciences Berlin, Charitéplatz 1, 10117 Berlin, Germany \\ ${ }^{5}$ Excellence Cluster Science of Intelligence, Marchstr. 23, 10587 Berlin, Germany \\ * r.kempter@biologie.hu-berlin.de \\ ${ }^{\dagger},+$ equal contribution
}




\section{Supplementary figures}

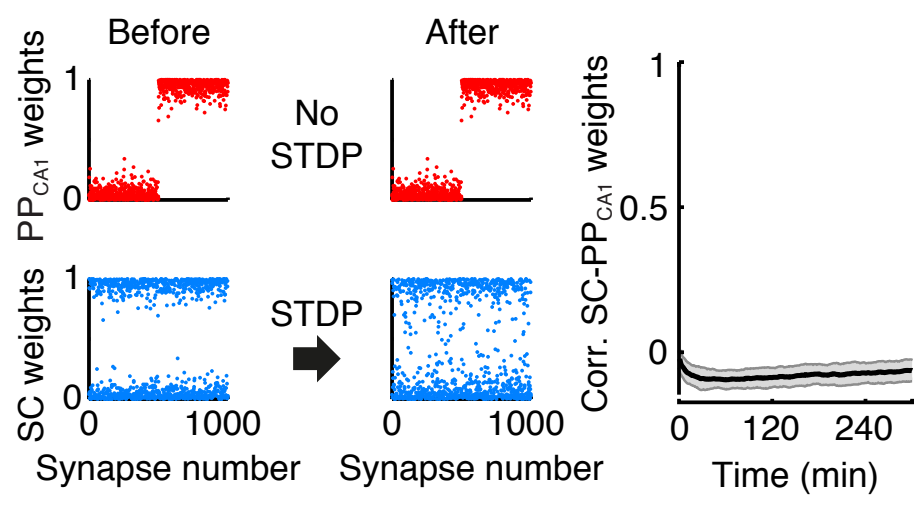

Supplementary Figure 1. Failure of consolidation of a synaptic weight pattern from non-plastic $\mathbf{P P}_{\mathrm{CA} 1}$ to plastic SC synapses. Panels as in Fig. 11E. Left and middle column: normalised synaptic weights before and after consolidation. Right column: time course of correlation between $\mathrm{SC}$ and $\mathrm{PP}_{\mathrm{CA} 1}$ weight vectors during consolidation (mean \pm SEM for 10 trials). 


\section{Theoretical analysis}

\section{STDP in a single cell with two input pathways.}

Let us first consider a single cell (which could be located in CA1) that receives inputs through two pathways (Fig. 1). We assume that memories, i.e., associations, are stored during the day in the weight vector $\mathbf{V}$ of the indirect path, and that consolidation occurs by transferring this information into the weights $\mathbf{W}$ of the direct path. For simplicity, we consider the case of a single rate-based neuron, which represents one of the output neurons in the simulated network. The spiking case of linear Poisson neurons leads to very similar results, apart from additional contributions from spike-spike correlations, which can be neglected for a large number of synapses (Kempter et al. 1999).

The output $y$ of the neuron is assumed to be given by a linear function of the input:

$$
y(t)=\mathbf{W}^{\top} \mathbf{x}(t)+\mathbf{V}^{\top} \mathbf{x}^{\prime}(t-D),
$$

where $\mathbf{x}$ and $\mathbf{x}^{\prime}$ denote the input arising from the direct and indirect pathway, respectively. We assume that the inputs $\mathbf{x}$ and $\mathbf{x}^{\prime}$ are related by some kind of (potentially nonlinear) statistical dependency, and that $\mathbf{x}^{\prime}$ arises from an indirect pathway and is therefore delayed by a time interval $D>0$. The notation is chosen such that the case where the two inputs to the two pathways are the same (apart from the delay) reduces to the condition $\mathbf{x}(t)=\mathbf{x}^{\prime}(t)$. This case corresponds to Fig. 1 of the main text. It is important to emphasise that in the following analysis of the learning dynamics, we consider the input arising during consolidation, e.g., during sleep, which may be statistically different from the input during memory storage or, more importantly, recall. If the correlation structure between the two pathways is different during consolidation and during recall, the consolidation process leads to a distortion of the memory in the sense that a different cue would be required to retrieve the memory. In the following, we will only consider the case where the correlation structure during consolidation is the same as during storage and recall.

We now consider the learning dynamics of a simple additive STDP rule that would 
result from a rate picture (neglecting spike-spike correlations; cf., Kempter et al. (1999)):

$$
\frac{\Delta \mathbf{W}}{T}=\eta \int_{-\infty}^{\infty} \mathrm{d} \tau \frac{1}{T} \int_{0}^{T} \mathrm{~d} t L(\tau) \mathbf{x}(t) y(t+\tau)=\eta \int_{-\infty}^{\infty} \mathrm{d} \tau L(\tau)\langle\mathbf{x}(t) y(t+\tau)\rangle_{T},
$$

where $L(\tau)$ is the learning window, which determines how much a pair of pre- and postsynaptic activity pulses (i.e., spikes) with a time difference $\tau$ changes the synaptic weight, and $\eta$ is a learning rate that scales the size of these changes. We adopt the convention that the time difference $\tau$ is positive when a presynaptic spike occurs before a postsynaptic spike. The notation $\langle\cdots\rangle_{T}=1 / T \int_{0}^{T} \cdots \mathrm{d} t$ indicates averaging over an interval of length $T$.

We assume that the integration time window $T$ can be chosen such that the weights do not change significantly during the integration time (i.e., a small learning rate), but that the statistics of the input are sufficiently well sampled so that boundary effects in the temporal integration are negligible. We also assume that the statistics of the inputs $\mathbf{x}$ and $\mathbf{x}^{\prime}$ are stationary, i.e., they do not change over time. Under these assumptions, we can insert the output firing rate from Eq. (39) into the learning rule and get

$$
\begin{aligned}
\frac{\Delta \mathbf{W}}{T}= & \eta \int_{-\infty}^{\infty} \mathrm{d} \tau L(\tau)\left\langle\mathbf{x}(t)\left[\mathbf{W}^{\top} \mathbf{x}(t+\tau)+\mathbf{V}^{\top} \mathbf{x}^{\prime}(t+\tau-D)\right]\right\rangle_{T} \\
\approx & \eta\left[\int_{-\infty}^{\infty} \mathrm{d} \tau L(\tau)\left\langle\mathbf{x}(t) \mathbf{x}(t+\tau)^{\top}\right\rangle_{t}\right] \mathbf{W} \\
& +\eta\left[\int_{-\infty}^{\infty} \mathrm{d} \tau L(\tau)\left\langle\mathbf{x}(t) \mathbf{x}^{\prime}(t+\tau-D)^{\top}\right\rangle_{t}\right] \mathbf{V}
\end{aligned}
$$

where $\langle\cdots\rangle_{t}$ denotes the average over all times. Eq. 41] describes the dynamics of the weights $\mathbf{W}$ in the direct pathway, which is given by an interplay of the correlation structure within and between the two pathways as well as the shape of the learning window $L$. In the following, we study under which conditions this weight update generates a consolidation of the input-output associations stored in the weights $\mathbf{V}$ of the indirect pathway into the weights $\mathbf{W}$ of the direct pathway. 


\section{Learning dynamics implement memory consolidation by a linear regression.}

In general, the learning dynamics is hard to analyse if the covariance matrices $\left\langle\mathbf{x}(t) \mathbf{x}(t+\tau)^{\top}\right\rangle_{t}$ and $\left\langle\mathbf{x}(t) \mathbf{x}^{\prime}(t+\tau-D)^{\top}\right\rangle_{t}$ are arbitrary objects. A case that can be studied analytically is that of separable statistics in which each of the two correlation matrices can be written as a product of a scalar function of the delay $\tau$ (here we use $f(\tau)$ and $g(\tau))$ and the covariance matrices for zero delay (denoted by $\left\langle\mathbf{x x}^{\top}\right\rangle$ and $\left\langle\mathbf{x} \mathbf{x}^{\prime \top}\right\rangle$, in which we omitted, for simplicity, the lower index $t$ ). In other words, we assume $\left\langle\mathbf{x}(t) \mathbf{x}(t+\tau)^{\top}\right\rangle_{t}=\left\langle\mathbf{x} \mathbf{x}^{\top}\right\rangle f(\tau)$ and $\left\langle\mathbf{x}(t) \mathbf{x}^{\prime}(t+\tau)^{\top}\right\rangle_{t}=\left\langle\mathbf{x} \mathbf{x}^{\prime \top}\right\rangle g(\tau)$. Note that this separability assumption is consistent with the simulations in Figs. 1, 3, and 4 of the main text. It is inconsistent with sequence replay during consolidation, because the time-delayed covariance of different place cells then depends on the relative spatial location of their place fields. The separable case is nevertheless an instructive scenario that provides insights into the dynamics of the consolidation process. For separable input statistics, the learning dynamics can then be simplified to

$$
\frac{1}{\eta} \frac{\Delta \mathbf{W}}{T}=\underbrace{\left[\int_{-\infty}^{\infty} \mathrm{d} \tau L(\tau) f(\tau)\right]}_{=: A}\left\langle\mathbf{x x}^{\top}\right\rangle \mathbf{W}+\underbrace{\left[\int_{-\infty}^{\infty} \mathrm{d} \tau L(\tau) g(\tau-D)\right]}_{=: B}\left\langle\mathbf{x x}^{\prime \top}\right\rangle \mathbf{V}
$$

If the scalar constant $A$ is negative (see below for conditions when this is the case), the learning dynamics is stable and converges to a unique fixed point that is given by

$$
\mathbf{W}=-\frac{B}{A}\left[\left\langle\mathbf{x x}^{\top}\right\rangle\right]^{-1}\left\langle\mathbf{x x}^{\prime \top}\right\rangle \mathbf{V}
$$

Note that apart from the factor $-\frac{B}{A}=: \beta$, this fixed point has the same structure as the closed-form solution of a linear regression. In fact, it is straightforward to show that the learning dynamics in Eq. 42 performs a gradient descent on the error function

$$
E(\mathbf{W}):=\left\langle\left(\mathbf{W} \cdot \mathbf{x}-\beta \mathbf{V} \cdot \mathbf{x}^{\prime}\right)^{2}\right\rangle_{t}
$$

If the scalar constant $B$ - and thus $\beta$ - is positive, the learning dynamics in the direct path converges to a weight configuration, for which the input $\mathbf{W} \cdot \mathbf{x}$ from the direct 
path is an optimal linear approximation of the input $\mathbf{V} \cdot \mathbf{x}^{\prime}$ from the indirect path, in pathway would contribute more to a potential recall than the original memory trace in the indirect pathway.

In terms of systems memory consolidation, the weights $\mathbf{V}$ of the indirect path change as new memories are acquired, so the fixed point in Eq. (43) for the weights W of the direct path is usually never reached. If it were, the direct pathway would merely represent a copy of the memories that are currently stored in the indirect path, rather than retaining older memories, as intended. The time scale of the learning dynamics of the direct path should therefore be longer than the memory retention time in the indirect path. In this case, the transient dynamics of the system is more important for the consolidation process than the fixed point.

Another important aspect to emphasise is that the consolidation is influenced by the correlation structure $\left\langle\mathbf{x} \mathbf{x}^{\prime \top}\right\rangle$ between the pathways that is encountered during the consolidation period. Intuitively, consolidation is achieved by matching the input $\mathbf{V} \cdot \mathbf{x}^{\prime}$ that is caused by "cues" $\mathbf{x}$ in the indirect path with the input $\mathbf{W} \cdot \mathbf{x}$ caused by the associated "cues" $\mathrm{x}^{\prime}$ in the direct path. In order for the consolidated memories to be accessible during recall, the relation between the "cues" in the two pathways (i.e., the correlation $\left\langle\mathbf{x x}^{\prime \top}\right\rangle$ between the two pathways) should be the same during recall as during consolidation. In terms of the hippocampus, this generates the prediction that during hippocampal replay, activity in layer III of entorhinal cortex should replay the same locations replayed in hippocampus proper, but represented in terms of the spatial coding scheme in the entorhinal cortex (i.e., grid cells).

The objective function argument in Eq. 44 only holds when the constant $A$ is negative. For positive $A$, the learning dynamics suffers from the common Hebbian instability and thus has to be complemented by a weight-limiting mechanism. The choice of this weight limitation (e.g., subtractive or divisive normalisation, weight bounds) will then have an impact on the dynamics and the fixed point of the learning process (Miller and MacKay, 1994, Dayan and Abbott, 2001). For the simulations in the main text, the parameters were always chosen such that the learning dynamics were stable $(A<0)$. Although this suggests that no weight limiting mechanism was required in principle, upper and lower bounds for the weights were nevertheless used, with no 


\section{Effects of temporal input statistics: Speed-up of input activity improves consolidation.}

The constants $A$ and $B$, which were defined in Eq. 42 as $A:=\int \mathrm{d} \tau L(\tau) f(\tau)$ and

$B:=\int \mathrm{d} \tau L(\tau) g(\tau-D)$, play an important role for the learning dynamics. The sign of

$A$ determines the stability of the learning dynamics, while $B$ should be positive to

obtain memory consolidation. For negative $B$, memories in the indirect path are

actively deleted from the direct path rather than consolidated therein. Both of these

constants depend on the interplay between the learning window $L$ and the temporal

input statistics, characterised by the functions $f$ and $g$. For the assumed separable

statistics, the function $f$ is fully determined by the autocorrelation function of the input

in the direct path and is therefore symmetric in time $\tau$.

A first interesting observation is that for an antisymmetric learning window $L$, the constant $A$ vanishes for symmetry reasons, $A=0$. Mathematically, this implies that the first term of the learning dynamics in Eq. 42 - the dependence on the weights in the direct path — vanishes. The weight update is then purely determined by activity arising from the indirect pathway. Intuitively, the balance of potentiation and depression in an antisymmetric learning window implies that the direct path, although able to drive the postsynaptic neuron, is causing equal amounts of potentiation and depression in all of its synapses. On average, synaptic changes are caused only by the indirect pathway, which therefore acts as a supervisor for the learning dynamics in the direct path. A thorough analysis under which conditions STDP can be used for supervised learning has been provided elsewhere (Legenstein et al., 2005, Pfister et al., 2006), and the results of this analysis are applicable in the present case. Functionally, the depressing part of the STDP learning window serves to neutralise the impact of the direct pathway on its own learning dynamics, effectively creating a supervised learning scenario.

Another interesting factor that determines the magnitude of the terms $A$ and $B$ is the time scale on which the inputs change (reflected, e.g., in the time constants of the correlation functions $f$ and $g$ ). Let us assume that both correlation functions $f$ and $g$ are maximal for $\tau=0$ and that they decay for large $|\tau|$, conditions that are reasonable 

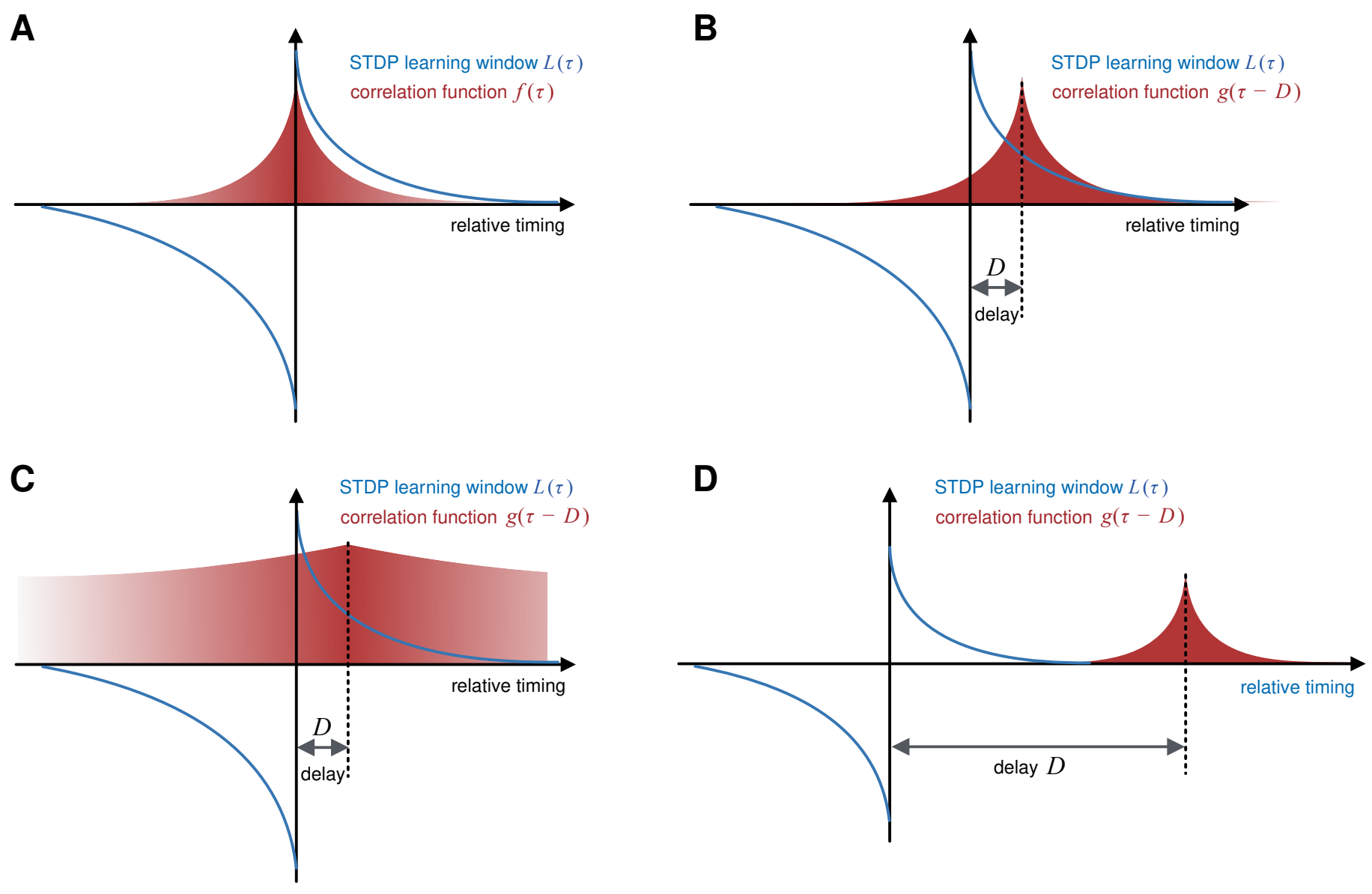

Supplementary Figure 2. Interaction of temporal correlations and the STDP learning window. The degree to which the weight dynamics of the direct path is driven by input from the direct and indirect pathways, respectively, is determined by the integrated products of the learning window $L(\tau)$, which is a function of the "relative timing" $\tau$, and the correlation functions $f$ (autocorrelation of inputs in the direct pathway) and $g$ (cross-correlation between input from the direct and indirect pathways). (A) For separable statistics, the autocorrelation function $f$ in the direct pathway is symmetric. If the learning window $L$ is antisymmetric, the integrated product (coefficient $A$ in Eq. 42 ) vanishes for symmetry reasons, and learning is driven by input from the indirect pathway alone. Then, on average, postsynaptic activity driven by the direct pathway does not interfere with consolidation. (B) The indirect pathway primarily induces potentiation in the direct pathway (coefficient $B>0$ in Eq. 42 ), if i) the delay $D$ between the pathways is positive, ii) the learning window is positive for positive delays, iii) the time scale of the decay of cross-correlations is shorter than the delay $D$ and the width of the learning window. These three conditions favor consolidation. (C) If the cross-correlations decay on a time scale that is much longer than the width of the learning window and the delay $D$, the indirect pathway can drive both potentiation and depression, and consolidation is weaker (i.e., the coefficient $B$ is smaller) than for shorter correlations. (D) If the delay $D$ between the direct and the indirect paths is longer than the width of the learning window $L$, the indirect pathway cannot induce systematic changes in the weights of the direct pathway (coefficient $B \approx 0$ ) and consolidation is ineffective. 
for most correlation structures. We also assume that the learning window has the typical structure of potentiation for causal timing $(L(\tau)>0$ for $\tau>0)$ and depression for acausal timing $(L(\tau)<0$ for $\tau<0)$ Bi and Poo, 1998, Markram et al., 1997; Sjöström et al. 2008). The delay $D>0$ in the indirect path shifts the maximum of the cross-correlation function $g$ into the potentiating part of the learning window (Supplementary Fig. 2B-D), while the maximum of $f$ remains in the transition region of potentiation and depression (Supplementary Fig. 2A). The following observations can be made concerning the constant $B$ as defined by the integral in Eq. 42. If the cross-correlation function $g(\tau)$ has a narrow enough peak at $\tau=0$ (i.e., narrower than the time scale of the learning window and the delay $D), B$ is positive, suggesting that consolidation can occur. The sharp localization of the correlation function $g$ corresponds to rapidly changing input signals. On the other hand, if the decay time constant of the correlation function $g$ is large compared to that of the learning window (Supplementary Fig. 2 $\mathrm{C}$ ), the depressing component of the learning window has more impact and reduces the constant $B$ and thus the efficiency of consolidation. In the case where the learning window is dominated by depression (as in our simulations), $B$ can even get negative for large time constants of the correlation function $g$, abolishing consolidation altogether. In summary, this dependence of the consolidation process on the time constants of the correlation functions suggests that during consolidation, the time scale of the activity in the two input pathways should be on the order of the time scale of the learning window. For the concrete example of a cell in CA1, the direct input $\mathbf{x}$ corresponds to perforant path inputs from the entorhinal cortex, while the indirect input $\mathbf{x}^{\prime}$ corresponds to Schaeffer collateral input from CA3. The time constants of behavioral experiences are typically slower (e.g., rate changes of place or grid cells in CA3 \& entorhinal cortex during exploration) than the learning window, suggesting that a real-time replay of recent experiences would not provide a favorable substrate for the consolidation of memories. The observed speedup of replay events during sleep could thus functionally serve the purpose of enabling consolidation by matching the time scales of activity and plasticity.

A final observation is that the time delay along the indirect path should not be excessively long, i.e., it should be on the order of the time scale of the learning window. Otherwise, the delayed correlations between the two pathways are too large to be 
exploited by spike timing-dependent plasticity (Supplementary Fig. 2D). This will limit ${ }_{721}$ the ability to consolidate from very long indirect paths into shortcuts, e.g., during the $\quad{ }^{722}$ hierarchical consolidation scheme in Figure 4 in the main text.

\section{Hierarchical consolidation}

As outlined in the main text and illustrated in Fig. 4 the suggested consolidation mechanism can be hierarchically iterated and leads to power law forgetting when the learning rates in the various pathways are suitably chosen. To get a theoretical understanding of this behaviour, let us consider the architecture shown in the Supplementary Fig. 3A, which is a generalised version of Fig. 44. The network consists of a hierarchy of $N+1$ input layers and $N+1$ output layers. For mathematical simplicity, the network is assumed to be linear, and the representation in the input layers is assumed to be the same, i.e., the weight matrices between the input layers (indicated in black in Supplementary Fig. 3A) are all simply the identity matrix.

Similarly, we also assume that all weight matrices between the output layers are also the identity matrix. The mathematical derivations presented in the following can be generalised to arbitrary weight matrices both in the input and the output pathways, but we prefer to treat the simple case to avoid cluttered equations and to make the theoretical approach more accessible.

We assume that due to newly acquired memories during the day, the weight matrix $W_{0}(t)$ that represents the memory trace in the hippocampus is varying in time, with an exponentially decaying autocorrelation function with time constant $\tau_{\text {overwrite: }}$ : $\left\langle\operatorname{tr}\left(W_{0}(0)^{\top} W_{0}(t)\right)\right\rangle_{t} \propto \exp \left(-t / \tau_{\text {overwrite }}\right)$, where $\operatorname{tr}$ denotes the trace of a square matrix.

All other pathways that project from an input layer to an output layer are plastic according to STDP. To derive the learning dynamics for these pathways, we first have to calculate the activity $\mathbf{y}_{i}$ in the $i$-th output layer,

$$
\mathbf{y}_{i}(t)=\sum_{j=0}^{i} c_{i j} W_{j}^{\top} \mathbf{x}_{j}\left(t-D_{i j}\right)
$$

where $\mathbf{x}_{j}$ denotes the activity in input layer $j$ and $c_{i j}$ denote weighting factors that determine the impact of the $j$ th pathway, i.e. the indirect pathway via $W_{j}$, on output 
layer $i$. These weighting factors are needed, because we would like to keep the weight matrices on a similar scale, but avoid that the activity increases from one output region to the next, because more synaptic pathways converge onto "later" output layers. The symbol $D_{i j}=2 D(i-j)$ denotes the total delay that is accumulated on the connection from the $i$-th input layer to the $i$-th output layer that traverses the $j$-th direct

"shortcut" pathway, relative to the direct shortcut from input layer $i$ to output layer $i$. For simplicity, we assumed that all connections have the same delay $D$. In a very similar way as in Eq. (41), the learning dynamics of the weight matrix $W_{i}$ in the direct path can be written as

$$
\frac{\mathrm{d} W_{i}}{\mathrm{~d} t} \approx \frac{\Delta W_{i}}{T} \approx \eta_{i} \sum_{j=0}^{i} c_{i j}\left[\int L(\tau)\left\langle\mathbf{x}_{i}(t) \mathbf{x}_{i}^{\top}\left(t+\tau-D_{i j}\right)\right\rangle_{t} \mathrm{~d} \tau\right] W_{j}
$$

where $\eta_{i}$ denotes the learning rate for the $i$-th pathway. For simplicity, we will assume that the different components of the input signal vector $\mathbf{x}_{i}(t)$ are uncorrelated amongst each other, and have identical temporal autocorrelations that are also independent of the layer index: $\left\langle\mathbf{x}_{i}(t) \mathbf{x}_{i}^{\top}(t+\tau)\right\rangle_{t}=\mathbf{I} f(\tau)$, where $\mathbf{I}$ is the identity matrix. The learning dynamics then simplify to

$$
\frac{\mathrm{d} W_{i}}{\mathrm{~d} t} \approx \eta_{i} \sum_{j=0}^{i} c_{i j} A\left(D_{i j}\right) W_{j}
$$

with $A(D):=\int L(\tau) f(\tau-D) \mathrm{d} \tau$.

To measure the degree to which a memory trace that is stored in the weight matrix $W_{0}$ at time $t=0$ is still present in the $j$-th shortcut pathway at a later time $t$, we compare the weight matrix $W_{j}(t)$ at time $t$ to the weight matrix $W_{0}(0)$ at time $t=0$. We quantify the correlation of these two matrices by calculating the summed overlap of the column vectors:

$$
O_{i}(t)=\operatorname{tr}\left[W_{0}(0)^{\top} W_{i}(t)\right]
$$

Note that the overlaps $O_{i}(t)$ are real numbers, and that their temporal dynamics for the shortcut connections (i.e., for all $i>0$ ) are dictated by the dynamics of the weight matrices in the network: 


$$
\begin{aligned}
\frac{\mathrm{d}}{\mathrm{d} t} O_{i}(t) & =\operatorname{tr}\left[W_{0}(0)^{\top} \frac{\mathrm{d} W_{i}(t)}{\mathrm{d} t}\right] \\
& =\eta_{i} \sum_{j=0}^{i} c_{i j} A\left(D_{i j}\right) \operatorname{tr}\left[W_{0}(0)^{\top} W_{j}(t)\right] \\
& =\eta_{i} \sum_{j=0}^{i} c_{i j} A\left(D_{i j}\right) O_{j}(t) .
\end{aligned}
$$

To capture the exponential decay of the initially stored memories in the "hippocampal" weight matrix $W_{0}$ due to the storage of new memories, the set of dynamical equations is completed by

$$
\frac{\mathrm{d}}{\mathrm{d} t} O_{0}(t)=-\frac{1}{\tau_{\text {overwrite }}} O_{0}(t)
$$

Note that the dynamics of the overlaps $O_{i}$ form a linear dynamical system.

To show that this mathematical description shows a power-law behavior akin to the simulated system in Fig. 4 of the main text, we simulated the equations with the following parameter choices. Consistent with the exponential decay of the learning rates in the simulations, we chose the learning rates as $\eta_{i}=2^{-i}$. The weighting factors $c_{i j}$ were chosen based on the assumption that output layer $i$ (for $i>0$ ) receives a fraction $\alpha$ of its input from the output layer $i-1$ below, and a fraction $1-\alpha$ via its direct shortcut connection (associated with the weight matrix $W_{i}$ ). Taking into account that the signal reaching layer $i$ through shortcut connection $j$ traverses several of these weighting stages (see Supplementary Fig. 3A A), this choice yields $c_{i j}=(1-\alpha)^{j} \alpha^{i-j}$. Note that $\sum_{j=0}^{i} c_{i j}=1$, so the activity level in different output layers should be similar. Finally, we assume that each synaptic transmission generates a fixed delay $D$ and that the autocorrelation function $f(\tau)$ decays much more quickly than the STDP learning window. In this case, we can approximate $A\left(D_{i j}\right)=\exp \left(-2 D \frac{(i-j)}{\tau_{\mathrm{STDP}}}\right)$. We chose $\tau_{\mathrm{STDP}}=40 \mathrm{~ms}$ as the decay time of the STDP learning window for positive delays $\tau>0$ and $D=2 \mathrm{~ms}$. Potential factors in $A\left(D_{i j}\right)$ are absorbed in the learning rates. As shown in the Supplementary Fig. $3 \mathrm{~B}$, the maximum of the overlaps $O_{j}$ indeed shows a power law decay. 
A

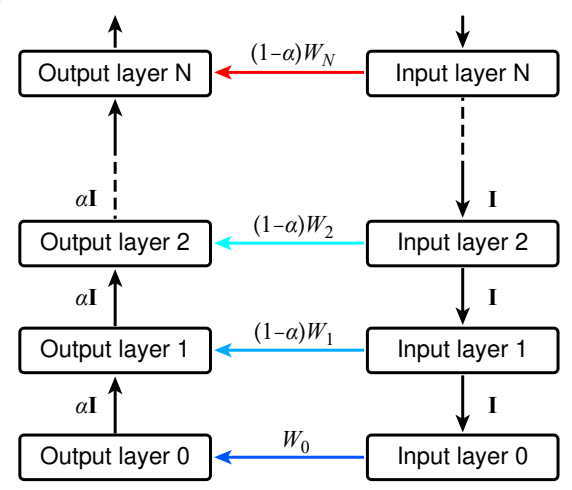

B

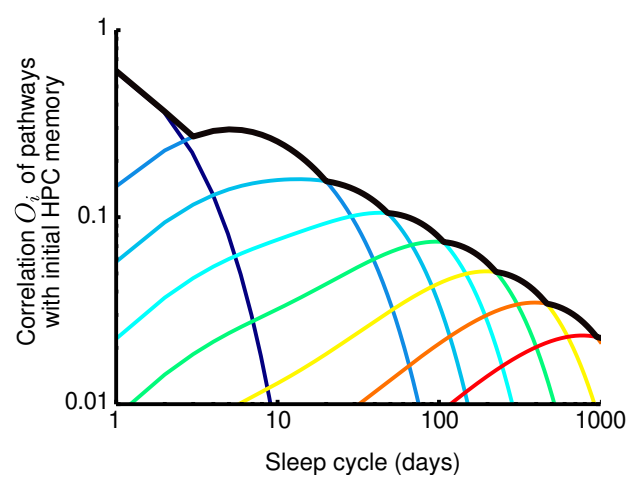

Supplementary Figure 3. Mathematical analysis of the hierarchical consolidation network. (A) The mathematical analysis is performed for a network consisting of $N+1$ input and $N+1$ output layers. All output layers (except output layer 0 ) weight the input from the previous layer with a factor $\alpha$ and the input via the shortcut pathway with a factor $1-\alpha$, to ensure that activity does not rise as increasingly many pathways converge onto the output layers. Input layer $i$ is hence connected to output layer $i$ through a shortcut connection with weight matrix $(1-\alpha) W_{i}$ (except for the bottom-most layers $i=0$, for which no factor $1-\alpha$ is required). All connections between input layers are set to the identity matrix $\mathbf{I}$, and all connections between output layers are set to $\alpha \mathbf{I}$, for notational simplicity in the derivations. The math can be generalized to arbitrary connection matrices, as long as the network is linear. Each connection introduces a synaptic delay of $D$. The multi-synaptic pathway from input layer $i$ to output layer $i$ via shortcut connection $j \neq i$ has a total delay of $(2(i-j)+1) \cdot D$, so the difference in delays between the pathway through shortcut $i$ and shortcut $j$ is $D_{i j}=2(i-j) \cdot D$. (B) The similarity $O_{i}$ of the weight matrix $W_{0}$ (in which memory traces are initially stored) and the shortcut connection $W_{i}$ as a function of the time elapsed after storage (colored lines), and their maximum (black line). Simulations shown for $D=2 \mathrm{~ms}, \alpha=0.8, \eta_{i}=2^{-i}$ and STDP time constant $\tau_{\mathrm{STDP}}=40 \mathrm{~ms}$. 


\section{References}

Bi, G.-q., and M.-m. Poo, Synaptic modifications in cultured hippocampal neurons: dependence on spike timing, synaptic strength, and postsynaptic cell type, Journal of Neuroscience, 18, 10,464-10,472, 1998.

Dayan, P., and L. F. Abbott, Theoretical Neuroscience, MIT Press, Cambridge, 2001.

Kempter, R., W. Gerstner, and J. L. van Hemmen, Hebbian learning and spiking neurons, Physical Review E, 59(4), 4498-4514, 1999.

Legenstein, R., C. Naeger, and W. Maass, What can a neuron learn with spike-timing dependent plasticity, Neural Computation, 17(11), 2337-2382, 2005.

Markram, H., J. Lübke, M. Frotscher, and B. Sakmann, Regulation of synaptic efficacy by coincidence of postysnaptic APs and EPSPs, Science, 275(5297), 213-215, 1997.

Miller, K. D., and D. J. C. MacKay, The role of constraints in hebbian learning, Neural

Pfister, J.-P., T. Toyoizumi, D. Barber, and W. Gerstner, Optimal learning, Neural Computation, 18(6), 1318-1348, 2006.

Sjöström, P. J., E. A. Rancz, A. Roth, and M. Häusser, Dendritic excitability and synaptic plasticity, Physiological Reviews, 88(2), 769-840, 2008. 